\title{
Technology Requirements of Exploration beyond Neptune by Solar Sail Propulsion
}

\author{
Malcolm Macdonald ${ }^{*}$, Colin $\mathrm{M}^{\mathrm{c}}$ Innes, ${ }^{\dagger}$ Gareth Hughes*, \\ University of Strathclyde, Glasgow, G1 1XJ, Scotland, E.U.
}

This paper provides a set of requirements for the technology development of a solar sail propelled Interstellar Heliopause Probe mission. The mission is placed in the context of other outer solar systems missions, ranging from a Kuiper Belt mission through to an Oort cloud mission. Mission requirements are defined and a detailed parametric trajectory analysis and launch date scan performed. Through analysis of the complete mission trade space a set of critical technology development requirements are identified which include an advanced lightweight composite High-Gain Antenna, a high-efficiency Ka-band travellingwave tube amplifier and a radioisotope thermoelectric generator with power density of approximately $12 \mathrm{~W} / \mathrm{kg}$. It is also shown that the Interstellar Heliopause Probe mission necessitates the use of a spinning sail, limiting the direct application of current hardware development activities. A Kuiper Belt mission is then considered as a pre-curser to the Interstellar Heliopause Probe, while it is also shown through study of an Oort cloud mission that the Interstellar Heliopause Probe mission is the likely end-goal of any future solar sail technology development program. As such, the technology requirements identified to enable the Interstellar Heliopause Probe must be enabled through all prior missions, with each mission acting as an enabling facilitator towards the next.

Associate Director, Advanced Space Concepts Laboratory, Department of Mechanical Engineering. malcolm.macdonald.102@strath.ac.uk, Associate Fellow of the AIAA.

$\dagger$ Director, Advanced Space Concepts Laboratory, Department of Mechanical Engineering. colin.mcinnes@strath.ac.uk, Member of the AIAA.

* Visiting Researcher, Advanced Space Concepts Laboratory, Department of Mechanical Engineering. 


\section{Nomenclature}

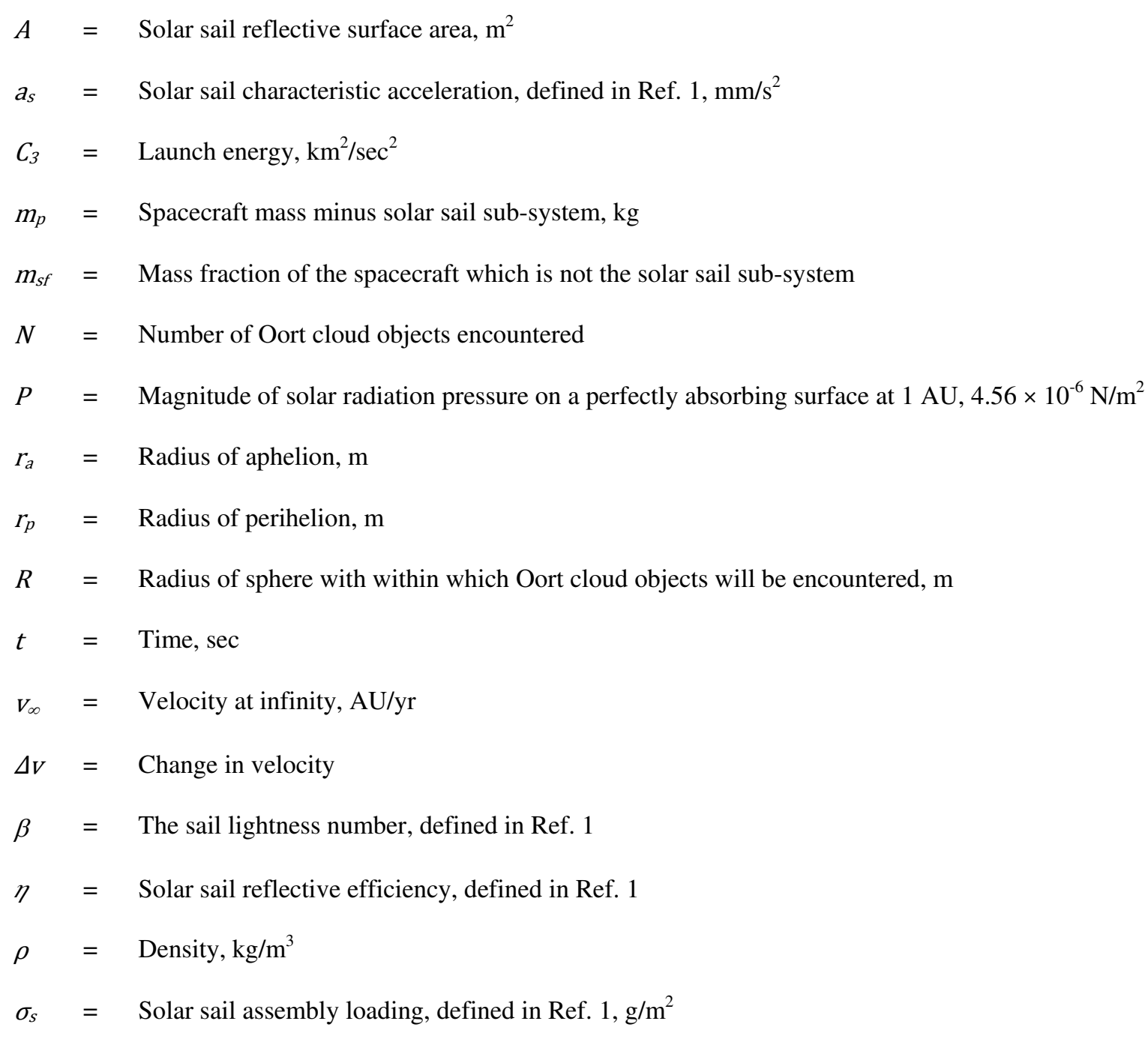

\section{Introduction}

$\mathrm{A}^{\mathrm{S}}$ the Voyager spacecraft continue towards interstellar space, and with the launch of the New Horizons spacecraft in January 2006, the concept of a dedicated mission for the in-situ exploration of the Heliopause boundary and interstellar space, up to $200 \mathrm{AU}$, is re-emerging within the science community. It is important to define and prepare critical technologies far in advance of novel science missions, ensuring the technologies are developed in a timely manner and that associated cost, risk and feasibility of potential future mission concepts can be properly estimated. Herein, the development of a set of requirements for the technology development of a solar sail Interstellar Heliopause Probe (IHP) mission are considered far before one is proposed by the scientific community. The IHP mission, and the technology requirements, are thereafter placed in the context of other outer solar systems missions, ranging from a Kuiper Belt mission through to an Oort cloud mission. 
Investigation of the Heliopause boundary would expand knowledge of the nature of the interstellar medium and its interaction with the solar wind. Such a mission would also be a precursor to the migration of deep space exploration beyond the solar system through the Oort cloud and on into true interstellar space. An IHP mission is, from a trajectory, propulsion and spacecraft systems viewpoint, a demanding endeavor to achieve within a reasonable timescale. Long cruise times must be endured, with the thermal environment ranging from close solar passes to deep space. Sufficient power generation and telecommunications downlink data rates at extreme distances from the sun are also crucial, as is science-driven autonomy.

A significant quantity of work in the past decade has been performed to assess the problem of trajectory design of an Interstellar Heliopause Probe, with a particular focus of this work looking at the application of solar sail technology and the design of solar sail trajectories 1 14. Additionally, the development of solar sail technology for outer solar system applications has been considered 15 17. Trajectory design for alternative propulsion systems, such as Solar and Nuclear Electric Propulsion (SEP and NEP) has also been considered 18, 19. Latterly in addition to trajectory and propulsion considerations, effort has begun to include the systems design of interstellar probes, with a significant amount of effort considering SEP and NEP missions 19 24. Additionally, two independent solar sail based holistic mission studies are found within the literature, one directed from NASA-JPL 25 27 and one from ESA-ESTEC 28 30. It is of interest to note that the NASA-JPL directed IHP mission attempts to gain a solar distance of $200 \mathrm{AU}$ in 15 years, concluding that a spin-stabilized disc solar sail is required for such a requirement. Meanwhile, the ESA-ESTEC directed study attempts to gain a solar distance of $200 \mathrm{AU}$ in 25 years, concluding that a three-axis stabilized square solar sail can satisfy such a requirement 30 . Noting that the IHP mission is often highlighted as an exemplar far-term solar sail mission 31, the disparity in required sail architecture is of critical importance to the development of solar sail technology. It is highly unlikely that even a successful mid-term solar sailing mission, such as Solar Polar Orbiter (SPO) 32, using a three-axis stabilized square solar sail architecture would provide much, if any, confidence to then progress to a spin-stabilized disc solar sail architecture for an IHP mission. As such, for a far-term solar sail mission such as IHP to be enabled the preceding missions 31 34 must act as enabling facilitators and must therefore develop the sail architecture required for far-term missions such as IHP.

This paper, for the first time, considers the IHP mission in context with other solar sail outer solar system missions. The paper begins by considering the IHP mission and attempting to resolve the apparent discrepancy over the required sail architecture while also determining the key technology development requirements to enable the 
mission. Subsequently, the paper considers missions to the two other primary sites of scientific interest beyond the orbit of Neptune, the Kuiper Belt and the solar gravitational lensing effect at $550 \mathrm{AU}$, in-order to place the IHP mission into context. Finally, the paper briefly considers a mission to the Oort cloud, the final region of scientific interest within our solar system and the definitive demarcation between our solar system and true interstellar space.

\section{Science Objectives of the Interstellar Heliopause Probe}

The scientific objectives of any IHP mission have been covered in detail through previous studies 2530 , however a brief discussion of the Heliopause region is included here for context. The objective of the IHP is to explore the local interstellar medium and the interaction region with the Heliosphere, known as the Heliopause. The Heliopause is the boundary between the outward flowing solar wind (hot ionised gas $\sim 10^{5} \mathrm{~K}$ ), and the relatively cool interstellar medium of partially ionised interstellar gas 35. The Heliosphere has a bow shock, towards the solar apex, where the Heliosphere moves through the interstellar medium at a velocity of order $26 \mathrm{~km} / \mathrm{s}$ relative to the local interstellar cloud. This bow shock is expected to lie at a distance of between 110 and $200 \mathrm{AU}$, slightly beyond the Heliopause and before the Local Interstellar Medium (LISM), but the actual location is somewhat uncertain. The apex of the bow shock, sometimes defined as the nose of the Heliopause, is located at an ecliptic longitude of $254.5^{\circ}$ and latitude $7.5^{\circ} 35$. Note that an inner shell is expected to be formed at 80-100 AU by the solar wind terminal shock, which is a predicted standing shockwave formed by the slowing of the supersonic solar wind to a sub-sonic flow 35,36 . The primary scientific goals of the considered IHP mission are, to investigate the influence of the interstellar medium on the solar system (and vice-versa), to explore the nature of the interstellar medium and the outer solar system and to take measurements of the Heliopause region using field and particle instruments.

It is worth noting that a further area of interest is that of organic material abundances in the Heliopause region. Organic material is found in both the solar system as well as in the interstellar medium, but it is not known if these non-terrestrial organic materials have a similar origin. The IHP could also be used to investigate the phenomenon known as the hydrogen wall 37 , a pile-up effect of neutral hydrogen atoms caused by charge exchange collisions, leading to weak coupling between the neutral and ionised hydrogen in the Interstellar Medium.

\section{Mission Requirements and Architecture of the Interstellar Heliopause Probe}

The IHP mission requirements and goals are detailed in Table 1 and are largely derived from 28 30. It is seen that the mission requirements are tightly constrained, with mission duration, spacecraft mass and launch vehicle predetermined. It is of note that the upper limit on mission duration is 10 years longer than considered by the NASA- 
JPL studies 2527 . The upper feasible limit on mission duration is difficult to quantify, for example the Voyager spacecraft remain operational over three-decades since launch yet the primary mission of these spacecraft was approximately three and twelve years, for Voyager 1 and 2 respectively. However, both spacecraft have continued to provide scientifically interesting data and as such operations have continued. As per requirement 5 , the IHP mission will provide continuous science data from 5 AU onwards, thus it is anticipated that the spacecraft will provide scientifically interesting data from an early stage. However, the primary goal of the mission is measurement of the interstellar medium, which therefore necessitates a funding commitment over a much longer period than originally envisaged for the Voyager spacecraft.

\section{Architecture Trade-Off}

Within the tight mission requirements detailed in Table 1 several potential solar sail mission architectures can be envisaged. Requirement 5 in Table 1;s derived from the desire that science data collection cover as large as possible a solar radius, therefore the sail will be jettisoned at 5 AU. It should however be noted that by maintaining control of the sail post-jettison, perhaps through a secondary sail-specific on-board computer, it may be possible to infer the density of the local medium from observations of the sail deceleration.

Four top-level solar sail mission architectures are detailed in Table 2, it is noted that a close solar approach of less than $0.25 \mathrm{AU}$ is generally considered very challenging 1 17. Conventional polyimide sail substrates (thermal limit $\sim 520 \mathrm{~K}$ [1, $)$ with conventional aluminum and chromium coatings will not be able to survive the local environment at less than $0.25 \mathrm{AU}$ for any significant duration. Hence, a solar approach of less than $0.25 \mathrm{AU}$ is highly unadvisable due to the large thermal loads placed on both the sail and the spacecraft system.

\section{Table 1 IHP mission requirements and goals}

\begin{tabular}{lll}
\hline Identifier & Objective & Requirement / Goal \\
\hline 1 & IHP shall traverse 200 AU within 25 years of launch & Requirement \\
2 & IHP shall travel through the nose of the Heliopause (7.5 lat. $254.5^{\circ}$ long.) & Requirement \\
3 & IHP shall be launched on a vehicle of similar capabilities to the Soyuz-Fregat & Requirement \\
4 & IHP shall provide a telemetry rate of 200 bps from 200 AU & Requirement \\
5 & IHP shall continuously collect science data from 5 AU outwards & Requirement \\
6 & IHP will have a dry mass of 200 kg at 200 AU & Goal \\
7 & IHP instrument payload suite will be 15-20 kg & Goal \\
\hline \hline
\end{tabular}


Table 2 Solar sail IHP mission architectures

\begin{tabular}{lll}
\hline \hline Mission & Name & Architecture \\
\hline IHP $_{1}$ & Multi-Photonic & Use a low acceleration square sail and multiple photonic assists and/or a \\
& Assist IHP & Jupiter Gravity Assist. \\
$\mathrm{IHP}_{2}$ & Single Photonic & Use a higher acceleration sail to allow only a single photonic assist and a close \\
& Assist IHP & solar approach thermally limited to 0.25 AU. \\
$\mathrm{IHP}_{3}$ & Fast IHP & $\begin{array}{l}\text { Use a very high acceleration sail, a single photonic assist, and a close solar } \\
\text { approach thermally limited to } 0.25 \text { AU to reach 200 AU in }<<25 \text { years. }\end{array}$ \\
$\mathrm{IHP}_{4}$ & Sail + Chemical & Use positive launch $\mathrm{C}_{3}$ and gravity assists, if required, to reach $0.25 \mathrm{AU}$, prior \\
& IHP & to sail deployment at the minimum solar radius. \\
\hline \hline
\end{tabular}

The proposed mission architectures are broadly similar, however the variations between each are significant and each mission merits closer examination, prior to definition of the selected solar sail solution.

$\mathrm{IHP}_{1}$ : Multi-Photonic Assist

This architecture is envisaged to be suitable for a 3-axis stabilized square solar sail, with multiple photonic assists used to attain the requirements and goals defined in Table 2. The use of a square sail is important to consider as this sail architecture has formed the focus of much of the recent solar sail hardware development by both ESA

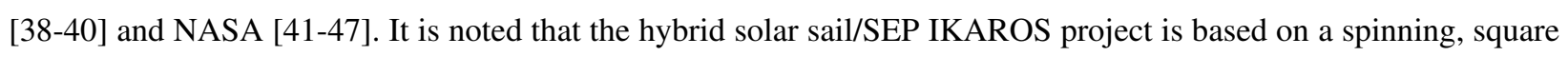
sail architecture 48 and could be a critical stepping stone towards the development of future pure solar sail technology. An advantage of a square solar sail is the likely availability of high turn rates during the close solar pass, also termed a solar photonic assist. A final consideration is the effect of multiple photonic assists, which may lead to increased optical surface degradation, although the impact of this is unclear 49. The 3-axis stabilized square solar sail is consider a lower performance sail due to the large structural mass required to support the sail reflective surface but which is negated through spin-induced forces on a spinning sail.

$\mathrm{IHP}_{2}$ : Single Photonic Assist

This architecture is envisaged to be suitable for a modest spinning disc solar sail, utilizing a single photonic assist to reduce optical surface degradation over $\mathrm{IHP}_{1}$. The likely drawbacks of this mission architecture are the relatively immature nature of spinning disc solar sail hardware and the reduced maneuverability of such a sail.

$\mathrm{IHP}_{3}$ : Fast

This architecture is very similar to the one considered by NASA-JPL 25 27], it will require a larger sail than $\mathrm{IHP}_{2}$ but will reduce the mission duration and hence operations cost. A notable drawback of this mission 
architecture is the increased Heliopause boundary transit velocity, which will negatively affect science data collection.

$\mathrm{IHP}_{4}$ : Sail + Chemical

This novel architecture is envisaged to consider the use excess launch $\mathrm{C}_{3}$ and unpowered gravity assists to reach 0.25 AU prior to sail deployment, hence maximizing the impact of solar sail propulsion while minimizing any impact of severe thermal aging on the sail performance. This architecture is analogous to an all-chemical mission, with the final, large chemical kick-stage replaced with a solar sail, with the required sail performance investigated to see if any advantage can be achieved over other mission architectures.

\section{Trajectory Design for the Interstellar Heliopause Probe}

Recall from Requirements 1 and 2 that the IHP is required to reach a solar radius of 200 AU in 25 years and pass through the nose of the Heliopause at a longitude/azimuth of $254.5^{\circ}$ and a latitude/elevation of $7.5^{\circ}$. As previously stated a vast quantity of work has been performed considering the design of solar sail trajectories for the IHP mission 1 1 14. However, it is difficult to gain clear conclusions from this previous work due to variations in trajectory design, id est, minimum allowable solar approach, and used solar sail propulsion model 1.,49. As such, a detailed parametric analysis is required to allow consideration of each of the mission architectures identified previously.

The NASA-JPL solar sail interstellar mission studies have focused largely on the use of high-performance spin-

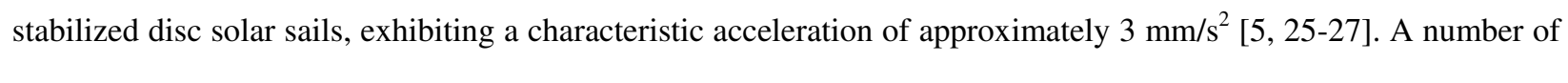
novel fast heliopause trajectories have also been presented, using a strategy of orbital angular momentum reversal, however this is not considered necessary for the IHP mission due to the level of sail performance required to enable such a trajectory 4. Note that parametric studies of multiple photonic assists have also been conducted by NASAJPL for missions to Pluto and the Kuiper belt, which could be extended to approximate the Heliopause scenario 7 .

Recent trajectories considered by Leipold, et al and ESA-ESTEC have considered lower performance sails to achieve a slower transit to $200 \mathrm{AU}$ with a conventional 3-axis stabilized square sail, with DLR (Deutsches Zentrum für Luft- und Raumfahrt; the German Centre for Air and Space Flight) derived composite booms 15,28 , 30 . In particular, a 21.2 year, $0.75 \mathrm{~mm} / \mathrm{s}^{2}$ ideal sail trajectory has been presented, that spirals down to $0.37 \mathrm{AU}$ and then executes a dual photonic assist, however the closest solar approach is just $0.1 \mathrm{AU}$, placing severe thermal loads on the spacecraft and sail assembly 15 . The mission scenario considered herein shall use the sail from Earth departure 
until it is jettisoned at $5 \mathrm{AU}$, as per requirement 5, and shall apply a strict solar approach limit of $0.25 \mathrm{AU}$ due to the thermal effects on the sail as discussed previously. Furthermore, due to thermal cycling issues where possible the trajectory design shall strive towards a single photonic assist to minimize optical surface degradation. Trajectories presented within this paper were developed using a sequential quadratic programming algorithm, NPOPT 6.2 50, along with the Accessibility and Deficit, or $\mathrm{A}^{\mathrm{n}} \mathrm{D}$ blending method of locally optimal control laws 114,51 .

\section{Parametric Trajectory Analysis}

For any level of sail performance, the solar photonic assist trajectory is optimized by minimizing the solar approach and maximizing the corresponding instantaneous radius of aphelion 14. As such any portion of the trajectory prior to the final close solar approach is simply seeking to maximize the instantaneous radius of aphelion while delivering the sail to the final close solar approach in as short a time as possible.

A parametric analysis was conducted through an approach of optimizing "half-arcs" from the final perihelion to $5 \mathrm{AU}$, as per Requirement 5. In this way, the effect of final close approach with low characteristic accelerations on trip time can be rapidly ascertained. Fixing the mission duration allows the available time to reach the final perihelion to be determined and the mission feasibility assessed. Figure 1 shows the time to $200 \mathrm{AU}$ from a range of final close approach radii at corresponding instantaneous aphelion values of 2 AU and 5 AU. Figure 1 was determined using a locally optimal energy gain control law which has been shown to give results very close to global optimal for such a scenario 14, 51. However, it should be noted that for this preliminary analysis the trajectory is simulated in two dimensions with an open final escape asymptote, such a simplification can result in a significant deviation from the time optimal, fully constrained, three-dimensional trajectory and as such is only valid as an initial analysis 14 .

It is noted that the perihelion velocity is relatively insensitive to the corresponding instantaneous radius of aphelion when perihelion lies in the range 0.05 to $0.4 \mathrm{AU}$ and aphelion 1 to $5 \mathrm{AU}$, with perihelion velocity varying by only $\pm 6 \%$. However, as seen in Figure 1 significant variations can occur once the trajectory is propagated to 200 AU, particularly at lower levels of sail performance and increased values of final close-approach radius. For example, an ideal sail providing a characteristic acceleration of $0.5 \mathrm{~mm} / \mathrm{s}^{2}$, with final solar close approach limited to $0.25 \mathrm{AU}$, will take approximately 175 years to reach $200 \mathrm{AU}$ if the instantaneous radius of aphelion was $2 \mathrm{AU}$ at the final solar close approach. However, if the instantaneous radius of aphelion is increased to $5 \mathrm{AU}$ the time to $200 \mathrm{AU}$ is reduced to approximately 43 years; a $75 \%$ reduction. Meanwhile, the variation for an ideal sail, providing a 
characteristic acceleration of $1.5 \mathrm{~mm} / \mathrm{s}^{2}$ drops by only $13 \%$. It is noted that using the velocity at 5 AU to approximate the trip time to $200 \mathrm{AU}$, assuming a constant velocity, is similarly flawed, with an error in the two previous scenarios of $260 \%$ and $10 \%$. Figure 1 allows determination of the available time to attain the final close solar approach for any given level of sail performance between $0.5 \mathrm{~mm} / \mathrm{s}^{2}$ and $1.5 \mathrm{~mm} / \mathrm{s}^{2}$ for missions to $200 \mathrm{AU}$. Note that Figure 1 assumes an ideal sail as defined in 11, a non-ideal sail can add between 5 and $10 \%$ to the trip time 10. From Figure 1, a $1.0 \mathrm{~mm} / \mathrm{s}^{2}$ sail with a minimum close solar approach of $0.15 \mathrm{AU}$ is required to reach close solar approach with a corresponding instantaneous radius of aphelion of 2 or 5 AU in approximately 1 or 5 years, respectively.

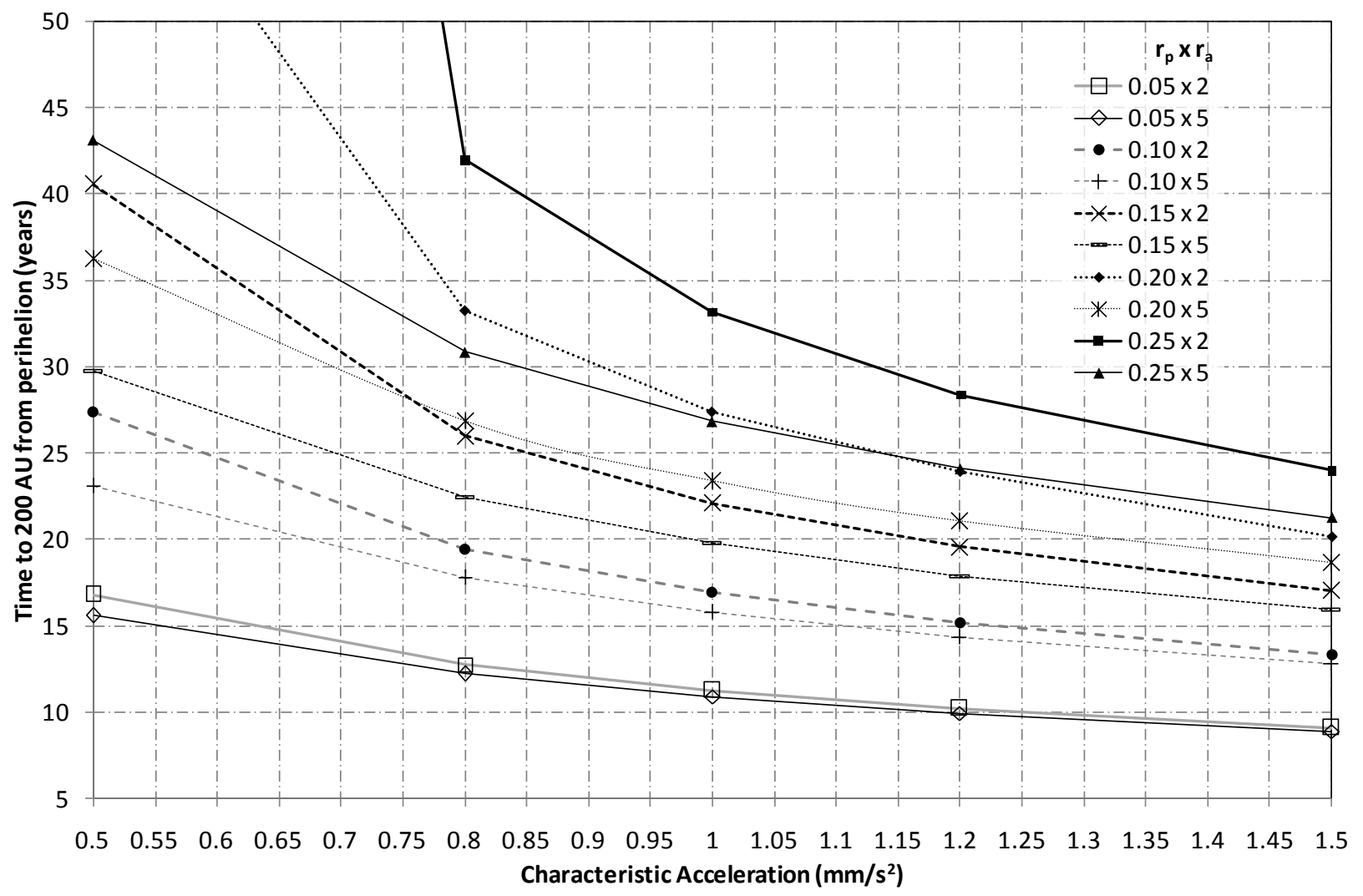

Figure 1 Closest approach to $200 \mathrm{AU}$ half-arc trajectory analyses

\section{Architecture Trade-Off}

Recall that within this mission scenario a strict thermal limit of $0.25 \mathrm{AU}$ is applied. Thus, from Figure 1 a $25-$ year half-arc trip time to $200 \mathrm{AU}$ with an instantaneous radius of aphelion of 2 or $5 \mathrm{AU}$ requires a sail characteristic acceleration of $1.44 \mathrm{~mm} / \mathrm{s}^{2}$ or $1.14 \mathrm{~mm} / \mathrm{s}^{2}$, respectively. Hence, sail characteristic accelerations above these values will be required to attain $200 \mathrm{AU}$ in less than 25 years from these orbits. For example, a sail characteristic 
acceleration of $1.3 \mathrm{~mm} / \mathrm{s}^{2}$ provides approximately -2 or +2.5 years to reach a close solar approach with a corresponding instantaneous radius of aphelion of 2 or $5 \mathrm{AU}$, respectively. Increasing sail performance to $1.5 \mathrm{~mm} / \mathrm{s}^{2}$ provides approximately 1 to 4 years to reach a close solar approach, depending on the corresponding instantaneous radius of aphelion. Therefore, with a perihelion limit of $0.25 \mathrm{AU}$ a characteristic acceleration of order $1.5 \mathrm{~mm} / \mathrm{s}^{2}$ appears necessary to meet Requirement 1 . This conclusion appears to contradict previous findings 30, which state that a solar sail with characteristic acceleration of $1.1 \mathrm{~mm} / \mathrm{s}^{2}$ can reach $200 \mathrm{AU}$ in 25 years, with a minimum solar approach of 0.25 AU. From Figure 1 it is noted however that such a solar sail would be required to reach the final close solar approach in between -1 and -6 years to enable a 200 AU trip in 25 years. However, if an instantaneous radius of aphelion of $5 \mathrm{AU}$ at close solar approach is assumed, along with constant velocity from 5 AU to $200 \mathrm{AU}$, then a period of 2.6 years is available to reach the final close solar approach. Similarly, increasing the instantaneous radius of aphelion to $10 \mathrm{AU}$ and making the same assumption provides a period of 3.6 years. As such, it appears possible that this previous work is based on an invalid assumption within the trajectory design.

The $\mathrm{IHP}_{3}$ architecture will clearly require a higher performance sail than $\mathrm{IHP}_{2}$ to enable a reduced trip time to $200 \mathrm{AU}$. As found in previous studies it was found that a sail characteristic acceleration of $3 \mathrm{~mm} / \mathrm{s}^{2}$ is required for a 15-year trip to $200 \mathrm{AU}$.

The mission architecture considered in $\mathrm{IHP}_{4}$ is highly novel and has not been previously considered within the literature. Within this architecture, the use of gravity assists and chemical propulsion was considered to reach the close solar approach prior to sail deployment, hence minimizing sail performance degradation prior to the vital solar photonic assist. Restricting the selection of the launch vehicle to a Soyuz-Fregat, as per Requirement 3, it was found that using only unpowered Jupiter gravity assists would deliver the probe to $0.25 \mathrm{AU}$ in approximately 9 years. Hence, from Figure 1 a very high-performance sail would be required to then reach $200 \mathrm{AU}$ within the 25 year target. It was found that a powered gravity assist at Venus $(\Delta \mathrm{v}=4.24 \mathrm{~km} / \mathrm{s})$ and a second at Jupiter $(\Delta \mathrm{v}=0.91 \mathrm{~km} / \mathrm{s})$ can enable an IHP mission with a sail characteristic acceleration of $2 \mathrm{~mm} / \mathrm{s}$. However, to gain a sufficient launch mass (assumed at $300 \mathrm{~kg}$ ) with the Soyuz-Fregat it was found that the kick-stage must have a specific impulse of order 500 seconds. It is thus concluded that mission architecture $\mathrm{IHP}_{4}$ can only enhance the IHP mission if Requirement 3 is relaxed and a larger launch vehicle used. 


\section{Reference Trajectory}

The primary technology driver of the IHP mission concept is clearly the solar sail. Therefore, mission architecture $\mathrm{IHP}_{2}$ was selected to minimize the technology requirements of the solar sail.

Using the $A^{n} D$ blending method of locally optimal control laws 14,51 a launch date scan was performed for an ideal sail with characteristic acceleration $1.5 \mathrm{~mm} / \mathrm{s}^{2}$. It should be noted that the $\mathrm{A}^{\mathrm{n}} \mathrm{D}$ method produces results which are typically within $2-3 \%$ of the global optimal 14, which will offset much of the anticipated error due to the assumption of an ideal sail with no optical surface degradation 10. The launch date scan is presented in Figure 2 for the year 2030, however it should be noted that the optimal launch will re-occur annually and hence this launch date is selected simply to facilitate trajectory analysis. The structure of the trajectory is a simple single solar photonic assist, as shown in Figure 3 where each of the trajectories within Figure 2 is seen. It is noted from Figure 2 and in Figure 3 that as the launch date moves through the period considered the required radius of aphelion passage decreases. It is anticipated that at some point a discontinuity will occur, resulting in the radius of aphelion passage increasing to a much larger value before decreasing in a fashion very similar to that shown in Figure 2 and in Figure 3. It is determined that the optimal launch date for an ideal sail with characteristic acceleration $1.5 \mathrm{~mm} / \mathrm{s}^{2}$ is 26 January, reaching $200 \mathrm{AU}$ in 23.17 years; the mission time line is detailed in Table 3.

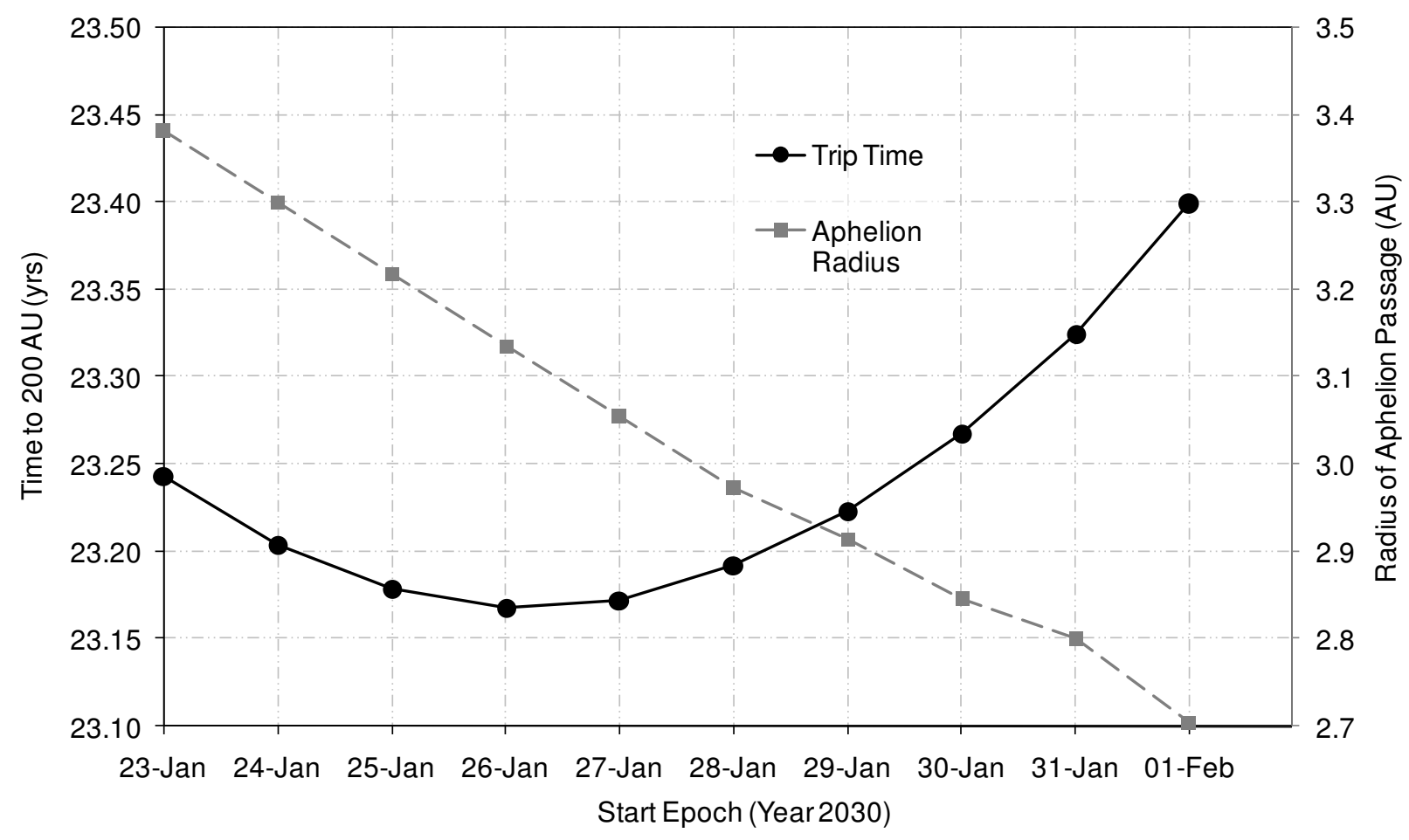

Figure 2 Launch date scan from 23 January until 01 February 


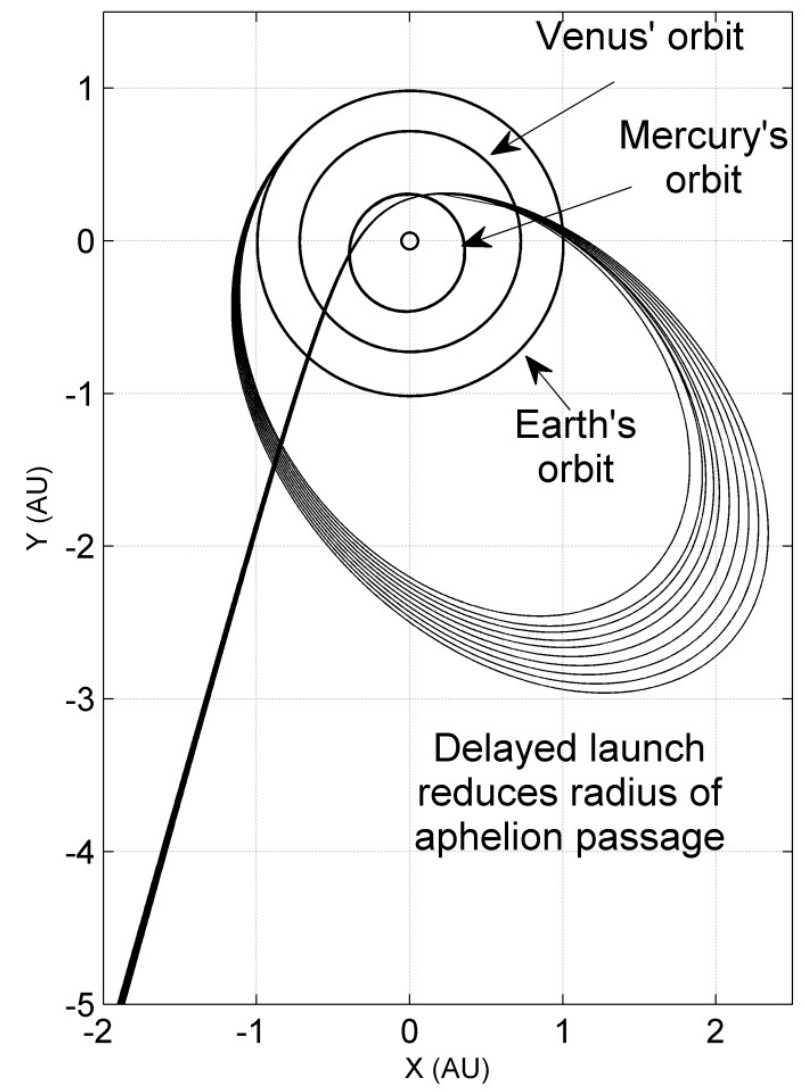

Figure 3 Trajectory plot over launch date scan from 23 January until 01 February, one trajectory per day

Table 3 IHP Reference Mission Timeline for 26 January launch

\begin{tabular}{ll}
\hline \hline Event & Time \\
\hline Launch & $\mathrm{T} 0$ \\
Aphelion passage & $\mathrm{T} 0+1.5 \mathrm{yrs}$ \\
Perihelion passage & $\mathrm{T} 0+2.8 \mathrm{yrs}$ \\
Sail Jettison (@5 AU) & $\mathrm{T} 0+3.2 \mathrm{yrs}$ \\
Kuiper Belt Transit $(40-55 \mathrm{AU})$ & $\mathrm{T} 0+5.7-8.3 \mathrm{yrs}$ \\
$100 \mathrm{AU}$ & $\mathrm{T} 0+12.9 \mathrm{yrs}$ \\
$200 \mathrm{AU}$ & $\mathrm{T} 0+23.2 \mathrm{yrs}$ \\
\hline \hline
\end{tabular}




\section{System Design for the Interstellar Heliopause Probe}

The focus of many previous solar sail IHP studies has been trajectory and sail design rather than system design. However, both the previous NASA-JPL $25 \mid 27$ and ESA-ESTEC $28 \| 30$ studies have touched on the issue through traditional system design methodologies. At this stage, it should be noted that within this paper the solar sail is simply considered a sub-system of the spacecraft, whereas traditionally the spacecraft is termed a payload of the sail. In order to identify the critical technology drivers for the IHP mission this study adopted a method of integrated statistical regression analysis, allowing the system design of the sail and the probe to be based on technology trends and for system interdependencies to be modeled through iteratively solved interdependencies. The integrated nature of the developed methodology allows key technology drivers and requirements to be identified more accurately and novel sensitivity analysis performed. For example, solar sail size or nominal mass versus radioisotope thermoelectric generator (RTG) power density, or data rate from $200 \mathrm{AU}$. It is this novel and integrated approach to system design which identified a critical turning point in required sail size for the SPO mission 32 and which is used to minimize the Advancement Degree of Difficulty (AD2) 33. of the mission being considered. For example, the most significant technology requirement for the IHP mission is clearly the solar sail which has a high AD2, thus if the solar sail technology requirements can be reduced by increasing the demand on other, more mature, technologies which have a lower AD2 then the overall mission AD2 is reduced.

Throughout the spacecraft design full redundancy is maintained wherever possible, however systems such as the high-gain antenna and many solar sail components, such as the sail film, cannot reasonably be supported by a backup system due to mass and/or volume constraints. The issue of systems redundancy on long duration missions is of critical importance and some significant work has recently been done in this area 52, 53. An overview of the spacecraft mass budget, based on a minimum solar approach of $0.25 \mathrm{AU}$, is presented in Table 4, where it is seen that the launch mass is $615 \mathrm{~kg}$, providing a Soyuz Fregat 2-1b launch mass margin of $1404 \mathrm{~kg}$. It should be noted that for a solar sail system design, unlike conventional system design, a large launch mass margin does not mean that the technology demands of the mission can be reduced simply by allowing the use of heavier components to "fill-up" the launch vehicle. As the launch mass margin is reduced the solar sail size will increase and hence the mission AD2 can actually be increased, therefore the goal of solar sail system design is to minimize the mission AD2; typically achieved by minimizing the solar sail size. Table 4 gives the current best estimate (CBE) mass, which then has a design maturity margin (DMM) added to give the total subsystem mass allocation. The DMM is 
added at equipment level, where $>5 \%$ is added for off-the-shelf items [European Cooperation for Space Standardisation (ECSS), category A/B], $>10 \%$ for off-the shelf items requiring minor modifications (ECSS category C), and $>20 \%$ is added for new design/development items, or items requiring major modifications or redesign (ECSS category D). Note in Table 4 that the added DMM can appear somewhat arbitrary. For example, the power subsystem DMM is $11 \%$ however this is simply a result of averaging the DMMs allocated at the equipment level.

Table 4 Mass budget, based on integrated statistical regression analysis

\begin{tabular}{|c|c|c|c|}
\hline System & CBE mass, kg & DMM, \% & CBE mass + DMM, kg \\
\hline Science Instruments & 25 & 0 & 25 \\
\hline Attitude and Orbit Control System (AOCS) & 6 & 5 & 7 \\
\hline Telemetry, Tracking and Command System & 55 & 8 & 62 \\
\hline On-Board Data Handling System & 1.5 & 10 & 2 \\
\hline Thermal \& Radiation & 14 & 10 & 16 \\
\hline Power & 50 & 11 & 56 \\
\hline Mechanism \& Structure & 27 & 15 & 31 \\
\hline Probe Nominal dry mass & & & 199 \\
\hline AOCS propellant, inc. sail separation allowance & 2.7 & 5 & 2.8 \\
\hline Probe Nominal wet mass & & & 202 \\
\hline Solar Sail Flight Components, see Table 5 & 83 & 33 & 110 \\
\hline $\begin{array}{l}\text { Solar Sail Jettisoned Components, inc. } \\
\text { deployment module, see Table } 5\end{array}$ & 163 & 18 & 201 \\
\hline Solar Sail nominal mass & & & 311 \\
\hline Nominal spacecraft launch mass & & & 513 \\
\hline System level margin, at $20 \%$ & & & 102 \\
\hline Total Mass at launch & & & 615 \\
\hline Soyuz Fregat 2-1b launch capacity & & & 2020 \\
\hline Launch margin & & & $1405(70 \%)$ \\
\hline
\end{tabular}


A $25-\mathrm{kg}$ allocation is provided for science instruments, which will most likely include a magnetometer. Hence, the spacecraft magnetic field must be low (preferably $<10^{-9} \mathrm{~T}$ ), known, and constant so that the science data can be corrected. From Table 4 it is seen that the probe wet mass is $202 \mathrm{~kg}$, this is the portion of the spacecraft which will perform the science operations of the mission once the sail has been jettisoned.

The science instruments require a spinning spacecraft, thus post sail jettison the probe will continue to spin. The primary attitude and orbit control system (AOCS) driver is the Ka-Band high gain antenna (HGA), which due to the high frequency of Ka-Band suffers from high losses at pointing accuracy worse than 180 arc seconds. The on-board telemetry, tracking and command (TT\&C) system contains two Ka-Band medium gain antennae (MGA), with a required pointing accuracy of 5400 arc seconds. The MGA is used for all communications during the period when the sail is attached to the IHP and for which a 10-m central cutout is provided in the sail surface. Post-sail jettison the HGA is used for primary communications. Therefore, during the mission phase when the IHP is attached to the solar sail pointing requirements are defined as 5400 arc seconds, which is achieved through the use of sun sensors. Star sensors are subsequently used once the sail is jettisoned and a much higher pointing accuracy is required. Pointing accuracy is maintained by a small cold-gas system once the sail has been jettisoned and by the sail prior to this. Note that a low gain S-band system is also included to provide robust communications prior to, and immediately post, sail deployment. The $2.5 \mathrm{~m}$ Ka-band HGA mass was found to be a key design driver due to a lack of sufficient data points on which to base the regression analysis. Many small HGA antennas have been flown, such that the HGA mass up to $2 \mathrm{~m}$ can be confidently project. However, beyond this only a limited dataset exists from which to project. Utilizing all the available data an exponential mass curve was developed which allows projection with some confidence up to a limit of $2.5 \mathrm{~m}$. This curve suggests a HGA mass of approximately $32 \mathrm{~kg}$. A secondary curve was then derived from this to project the mass of a more advanced lightweight composite HGA. This lightweight HGA was projected at approximately $24 \mathrm{~kg}$, a $25 \%$ mass saving. The development of such a lightweight composite HGA was determined to be a critical mission requirement, however it should also be noted that the mass and power requirements of the Ka-band travelling-wave tube amplifier within the TT\&C system is also of critical concern, with the current output power of $75 \mathrm{~W}$ towards the upper end of current technology.

The TT\&C sub-system is a critical mission driver and further study is required. Specifically, the use of optical communications should be investigated, which would require a considerable improvement in pointing accuracy. Additionally, issues such as the acquisition strategy would have to be re-addressed to account for the long slant 
ranges involved. The light travel time to $200 \mathrm{AU}$ is 26 hours, hence using a beacon system, as employed currently, would not be suitable over such long slant ranges. This is an issue that must be addressed for all outer solar system missions when considering optical communications. In addition to acquisition strategy, lightweight components and long lifetime lasers would need to be developed to make optical communications viable for the IHP mission. For these reasons a radio frequency link was assumed within this study; however, a more in-depth communications analysis is required prior to final architecture selection.

\section{Solar Sail Sub-System Design}

The primary function of the solar sail sub-system is to deploy, support and control a large, light-weight reflective surface which will impart a momentum onto the complete spacecraft assembly due to incident and reflected solar photons. Following optics-based reflectivity models it has previously been assumed that the sail film must be held in high-tension to ensure maximum reflectivity, such models assume symmetry about the surface normal beyond \pm 6 $10^{\circ}$ with a perturbed Lambertian distribution, seeking to achieve a specular reflection. Thus, scattered photons are seen as being of no use. A solar sail can however gain thrust from all reflected photons, diffusely scattered or not, indeed prior experimentation by NASA-JPL has shown that aluminum can provide a reflectivity of $0.88-0.90$ for flatness \pm 4.0 , thus requiring only low film tension 54. Furthermore, recent testing has shown that even heavily wrinkled (aluminum coated) material retains $88 \%$ reflectivity under zero tension 55 . As such, if the sail film is held in high-tension it is highly likely that excess mass is being deployed without adequate compensation through increased reflectivity.

It was determined previously that a characteristic acceleration of $1.5 \mathrm{~mm} / \mathrm{s}^{2}$ is required to attain the mission goals within the defined requirements. The mass fraction of the spacecraft which is not the solar sail sub-system is,

$$
m_{s f}=\left[1-\left(\frac{a_{s} \sigma_{s}}{2 \eta P}\right)\right]
$$

therefore, the required sail surface area for a given probe mass, $\mathrm{m}_{\mathrm{p}}$, can be determined from,

$$
A=\left[m_{p}\left(\frac{1}{m_{s f}}-1\right)\right] / \sigma_{s}
$$

It is seen from Eq. (1) that as the sail assembly loading is increased the mass fraction of the spacecraft which is not the solar sail sub-system decreases. Therefore, from Eq. (2) it is noted that as the sail assembly loading is increased the required sail reflective surface area will tend towards infinity, which in the scenario of this study occurs at a sail assembly loading of just over $5.168 \mathrm{~g} / \mathrm{m}^{2}$. Clearly a solar sail reflective surface area of infinity is not feasible. 
Therefore, initially considering a 3-axis stabilized square solar it is found that for a side-length of less than $300 \mathrm{~m}$ the sail assembly loading must be less than $3 \mathrm{~g} / \mathrm{m}^{2}$, which for a thermal limit of $0.25 \mathrm{AU}$ is very challenging 1 . Due to the clear difficulty of developing a 3-axis stabilized square solar sail, a spinning disc sail is considered, based on the NASA-JPL design 25 27,56. The spinning disc sail is scaled based on the other system masses, sail size and moment of inertia, while maintaining a constant sail fabric tension of $6925 \mathrm{~Pa}(\sim 1 \mathrm{psi})$. The absence of booms from the spinning disc sail allows for a significant reduction in sail assembly loading while also allowing the design to be generated without a significant level of prior technical data, enabling definition of technical requirements. The solar sail proposed for the NASA Interstellar Probe Mission is $410 \mathrm{~m}$ in diameter, with an assembly loading of $1 \mathrm{~g} / \mathrm{m}^{2}$. While there is nothing technically wrong with such a design it is considered quite aggressive due to the advanced nature of the required technologies along with the required sail size to achieve the mission goals in 15 years. Hence, design margins are added within this study, increasing the sail assembly loading to more attainable mid-term values and reducing the overall mission AD2. A spinning disc sail is not only tensioned by centripetal forces when fully deployed, but it must also be deployed using these spin-induced forces due to the absence of structural elements, which add mass, such as stiff booms. As such a dedicated deployment module, employing cold-gas thrusters mounted on $2.5 \mathrm{~m}$ booms to provide adequate torque for spin-up is required which is jettisoned following sail deployment. Control of the spinning disc sail is accomplished by sliding the centrally mounted payload along short rails to offset the centre-of-mass with respect to the centre-of- pressure, causing the sail spin axis to precess; this could also be accomplished by using a gimbaled boom.

From Figure 4, the sail areal density must be less than $1.6 \mathrm{~g} / \mathrm{m}^{2}$ to fit within the capacity of the launch vehicle, however this leaves zero margin for growth within other systems, it is also noted that at an areal density of $1.0 \mathrm{~g} / \mathrm{m}^{2}$ the mass of the deployment system exceeds that of the sail. Thus, from this sail design a technology requirement of a sail film areal density of $0.5 \mathrm{~g} / \mathrm{m}^{2}$ is derived to ensure sufficient technology margin for other sail systems and to ensure the sail size does not become a technology driver. The solar sail system in Table 4, Table 5 and Figure 4 corresponds to a sail with radius $139 \mathrm{~m}$, a centre cut-off of $10 \mathrm{~m}$ to allow a $4 \pi$ steradian field-of-view from the spacecraft and an assembly loading $1.8 \mathrm{~g} / \mathrm{m}^{2}$. A detailed breakdown of the sail and its deployment system mass is given in Table 5, where it is noted that the design point falls within the range of prior spinning sail design studies. 


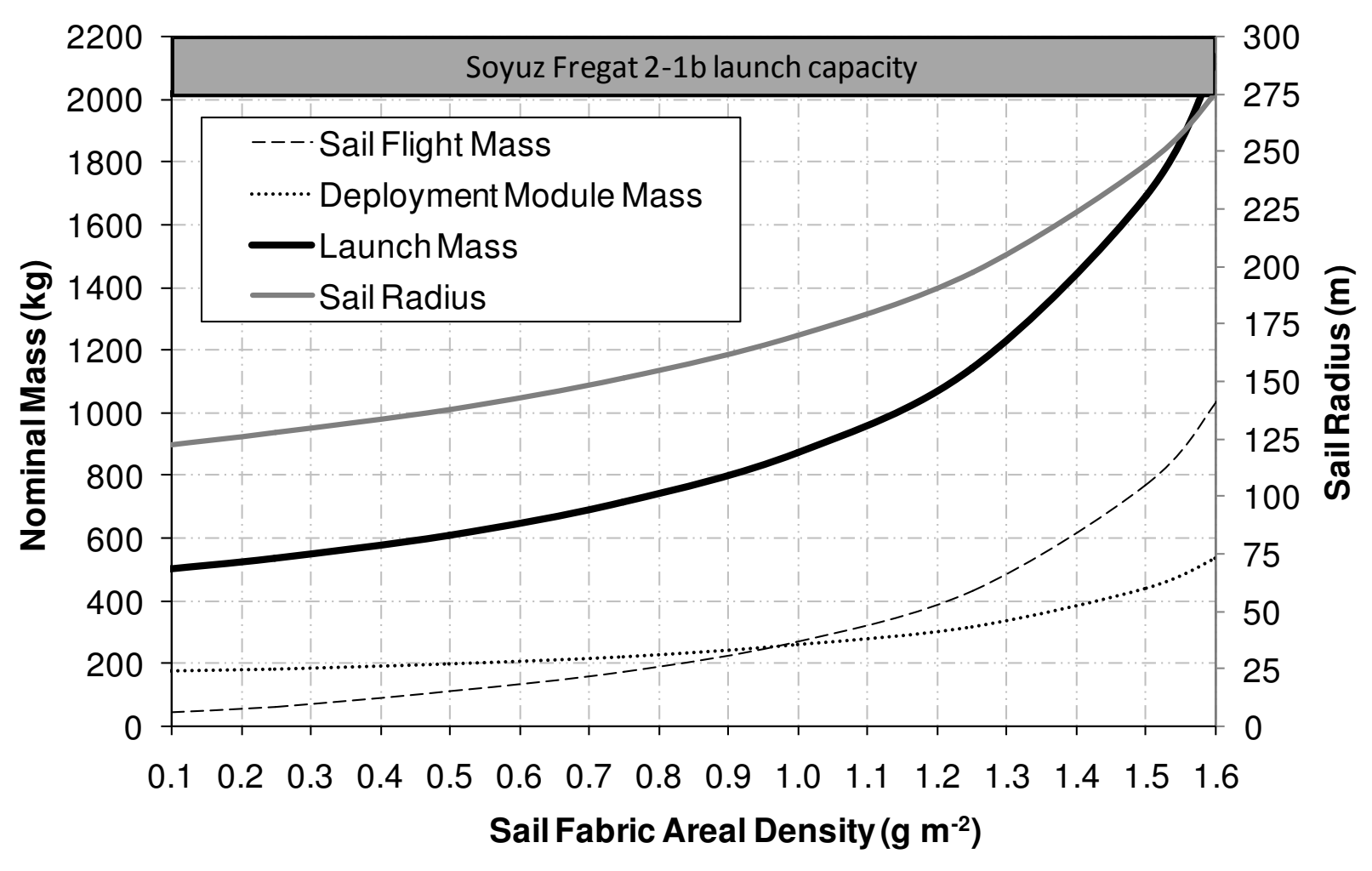

Figure 4 Effect of variations in sail areal density on sail size and mission mass budget 
Table 5 Mass budget, of solar sail based on integrated statistical regression analysis

\begin{tabular}{|c|c|c|c|}
\hline System & CBE mass, kg & DMM, \% & CBE mass + DMM, kg \\
\hline $\begin{array}{l}\text { Sail Fabric Mass } \\
\text { (areal density of } 0.5 \mathrm{~g} / \mathrm{m}^{2} \text { ) }\end{array}$ & 30 & 30 & 39 \\
\hline $\begin{array}{l}\text { Seaming tapes, Gore reinforcements } \\
\text { ( } 20 \% \text { of sail fabric mass) }\end{array}$ & 6 & 30 & 8 \\
\hline $\begin{array}{l}\text { Cylinders, Brackets, Braces, Rollers } \\
\text { ( } 25 \% \text { of sail fabric mass) }\end{array}$ & 8 & 30 & 10 \\
\hline Rim \& Tip Masses & 12 & 30 & 15 \\
\hline $\begin{array}{l}\text { Yoke plates, tether locks } \\
\text { (Fixed mass of } 6 \text { yoke plates and } 6 \text { locks) }\end{array}$ & 9 & 30 & 12 \\
\hline Gore deployment tethers & 3 & 40 & 4 \\
\hline Kevlar tether of radius $0.5 \mathrm{~mm}$ & & & \\
\hline Sail CofG/CofP offset system & 5 & 30 & 7 \\
\hline $\begin{array}{l}\text { Misc. structure } \\
\text { (10\% of total sail mass) }\end{array}$ & 10 & 40 & 15 \\
\hline Solar Sail Flight Components & & & 110 \\
\hline $2.5 \mathrm{~m}$ booms for RCS thrusters moment arm & 4.5 & 15 & 5 \\
\hline $\begin{array}{l}\text { Discarded Sail Structure } \\
\text { (areal density of } 1 \mathrm{~g} / \mathrm{m}^{2} \text { ) }\end{array}$ & 60 & 40 & 85 \\
\hline Cold Gas Spin-Up System & 33 & 10 & 37 \\
\hline Primary Structure & 30 & 15 & 34 \\
\hline Launch Release Mechanism & 15 & 15 & 17 \\
\hline $\begin{array}{l}\text { Launch Vehicle Interface } \\
\text { ( } 4 \% \text { of nominal launch mass) }\end{array}$ & 20 & 15 & 23 \\
\hline Solar Sail Jettisoned Components & & & 201 \\
\hline
\end{tabular}




\section{Power}

The design maximum average power load was found to be during telecommunication downlink periods, while science acquisition continues. The end-of-life (EOL) design value was found to be $266 \mathrm{~W}$, including design maturity margin and a $20 \%$ system level margin. In order to generate sufficient power a RTG based system was specified. The EOL power design value corresponds to a beginning-of-life (BOL) requirement of $325 \mathrm{~W}$, assuming a Plutonium-238 half-life of 86.4 years. Using this BOL power load the effect of RTG power density on IHP mass, sail size, sail mass and total launch mass can be analyzed. The system design assumes excess heat from the nuclear source can be utilized for thermal control. From Figure 5 it is seen that RTG power density does not have a notable impact on sail size. For a conventional RTG of the type used on Cassini or Galileo the sail radius is relatively modest, at only $160 \mathrm{~m}$ for a $5 \mathrm{~W} / \mathrm{kg}$ RTG. However, the required IHP mass is $255 \mathrm{~kg}$, considerably above the goal mass. A power density of approximately $12 \mathrm{~W} / \mathrm{kg}$ is required is to reduce the IHP mass towards the goal value, requiring a sail radius of approximately $138 \mathrm{~m}$. It is noted however that between $10-15 \mathrm{~W} / \mathrm{kg}$ the gradient of sail radius decreases such that it can be stated, for the design power load, a power density of above $12 \mathrm{~W} / \mathrm{kg}$ is not required as it will provide only modest benefits for the sail sizing. Current RTGs are approximately $7 \%$ efficient,

delivering about $5 \mathrm{~W} / \mathrm{kg}$ 57. The required power density corresponds to an Advanced Radioisotope Power Source (ARPS), based on $21 \%$ efficient AMTEC (alkali-metal thermoelectric converter) cells using a two-brick generalpurpose heat source (GPHS). 


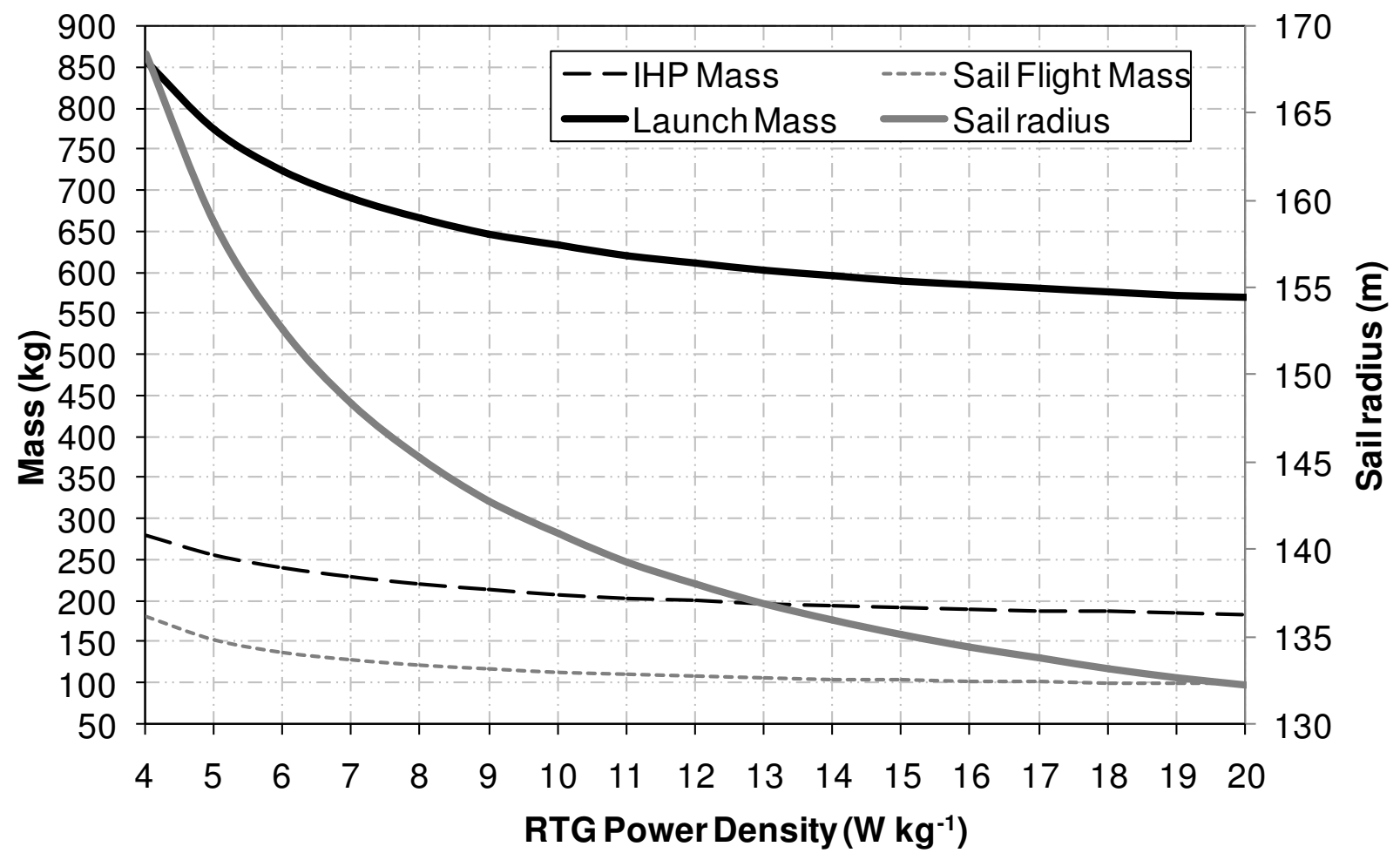

Figure 5 Effect of variations in RTG power density on sail size and mission mass budget

\section{Potential Mission Architecture Adaptations}

Originally the mission architecture was divided into four options, however on completion of the mission study it is prudent to review these architectures and consider possible adaptations. While noting the limited availability of such opportunities, a Jupiter gravity assist (JGA) was considered during the outgoing escape trajectory. It is immediately noted that the orbit inclination of Jupiter is 1.3 degrees [58, as such the 7.5 degree latitude of the Heliopause nose needs to be attained entirely through the gravity assist to ensure compliance with requirement two, i.e. that the probe travel through the nose of the Heliopause at $254.5^{\circ}$ longitude. There are, of course, additional operational risks associated with a close Jupiter swing-by, as well as the radiation issue of passing so close to Jupiter. Radiation exposure should however be minimal since the spacecraft will be travelling at high speed. The gravity assist was modelled as a simple patched-conic, assuming 2-body dynamics, with Jupiter at 5.2 AU from the sun. The attainable $\Delta v$ for pre-JGA velocities of between 5 and $15 \mathrm{AU} / \mathrm{yr}$ and for a range of perijove radii covering the mean distances of the Galilean moons was considered. It was found that using a JGA for fast Heliopause missions with characteristic accelerations greater than $1.5 \mathrm{~mm} / \mathrm{s}^{2}$ does not offer any significant reduction in trip 
time. However, low performance sail trajectories, which would ordinarily reach the Heliopause in over 25 years, would gain a modest benefit from a JGA. It is thus concluded that a JGA not be considered further at this stage of design, however an opportunistic flyby should not be ruled out.

From Table 4, a considerable launch mass margin is available. Therefore, an investigation of the effect of using the associated considerable excess $\mathrm{C}_{3}$ capacity was conducted. It can be anticipated that an excess $\mathrm{C}_{3}$ would offer only a small benefit to IHP missions, enabling more rapid arrival at the close solar approach. For low performance sails, where multiple spirals are needed to reach the final close solar approach, it is anticipated that the excess $\mathrm{C}_{3}$ may be used to reach the close solar approach in fewer solar revolutions. A launch excess energy of $20 \mathrm{~km}^{2} / \mathrm{s}^{2} \mathrm{was}$ considered. It was found, as expected, that for low performance sails the excess $\mathrm{C}_{3}$ may be used to reach the close solar approach in fewer solar revolutions. However, the actual time saving prior to sail jettison was minimal in these cases with the primary benefit actually a reduction in accumulated thermal loads. At the 5 AU sail jettison the cruise velocity, for a $0.85 \mathrm{~mm} / \mathrm{s}^{2}$ solar sail was found to be reduced by $0.83 \%$ against the optimal zero excess energy case. Thus, as a result of the reduced time to sail jettison and the reduced cruise velocity the complete mission duration is reduced by only $2 \%$. Increasing the sail performance to a zero excess launch energy, single revolution level, id est, a $1.5 \mathrm{~mm} / \mathrm{s}^{2}$ sail, it was found that the trajectory optimizer is unable to gain a close solar approach of $0.25 \mathrm{AU}$, instead using a close solar approach of 0.29 AU. Once again the initial spiral down time has been reduced, however due to the increased close solar approach and inability of the sail, due to this time reduction, to increase the instantaneous radius of aphelion the cruise velocity at sail jettison was found to be reduced by $29 \%$, with the resultant trip time to $200 \mathrm{AU}$ increased by $42 \%$. This clearly illustrates the importance of minimizing the radius of close solar approach. It thus seems clear that once the sail performance reaches a level where a zero excess launch energy, single revolution trajectory is enabled, any additional energy must be sufficiently large to enable both a close solar approach and for the instantaneous radius of aphelion to be sufficiently increased. Therefore, a terrestrial planet gravity-assist would also not provide sufficient additional energy to benefit the mission architecture.

To confirm this result a $3.0 \mathrm{~mm} / \mathrm{s}^{2}$ sail was considered using the available excess launch energy; it was found that at sail jettison, which occurred earlier than in the zero excess launch energy scenario, the cruise velocity was reduced by almost $10 \%$ with the trip time to $200 \mathrm{AU}$ reduced by less than $5 \%$. It is thus clear that excess launch energy is of little or no benefit within the mission scenario detailed within this paper, however it can be of modest benefit at extremes of sail performance. 


\section{A Kuiper Belt Fly-Through Mission}

Having considered a Heliopause mission, the less challenging scenario of a Kuiper Belt (or Edgeworth-Kuiper Belt) mission is briefly considered. Targeting a solar distance of between 30 and $55 \mathrm{AU}$, the Kuiper Belt in addition to being a scientifically worthwhile target in its own right, can reasonably be considered a technology pathfinder towards a Heliopause mission. For this mission, as previously, a system design using a method of integrated statistical regression analysis was performed which detailed a wet spacecraft mass, excluding solar sail, of $152.6 \mathrm{~kg}$. It was found that to reach the orbit of Pluto within 15 years a sail characteristic acceleration of $0.5 \mathrm{~mm} / \mathrm{s}^{2}$ was required. Thus, directly adapting the sail defined for the IHP mission, the required sail radius is $63.2 \mathrm{~m}$, with a sail assembly loading of $3.3 \mathrm{~g} / \mathrm{m}^{2}$. The solar sail flight component mass was found to be $40.7 \mathrm{~kg}$ with a jettisonable mass of $121.4 \mathrm{~kg}$, giving a total launch mass, including a final $20 \%$ system level margin, of $377.6 \mathrm{~kg}$. The mission launch mass is relatively low, enabling the low cost Rockot launch vehicle to be used, further reducing mission cost from conventional missions which require much larger and more expensive launch vehicles. It should be noted that this mission can be performed by a 3-axis stabilized square sail of side length $130 \mathrm{~m}$ and assembly loading $6.5 \mathrm{~g} / \mathrm{m}^{2}$, however such a mission could not easily act as a technology pathfinder towards a Heliopause mission due to the required sail performance of the later mission. The Kuiper Belt mission timeline is detailed in Table 6.

\section{Table 6 Kuiper Belt mission timeline}

\begin{tabular}{ll}
\hline \hline Event & Time \\
\hline Launch & $\mathrm{T} 0$ \\
$1^{\text {st }}$ perihelion passage & $\mathrm{T} 0+0.5 \mathrm{yrs}$ \\
$2^{\text {nd }}$ perihelion passage & $\mathrm{T} 0+1.75 \mathrm{yrs}$ \\
$3^{\text {rd }}$ perihelion passage & $\mathrm{T} 0+4.6 \mathrm{yrs}$ \\
Arrival at Kuiper Belt & $\mathrm{T} 0+14.1 \mathrm{yrs}$ \\
End of primary mission at $50 \mathrm{AU}$ & $\mathrm{T} 0+20 \mathrm{yrs}$ \\
End of secondary mission at $100 \mathrm{AU}$ & $\mathrm{T} 0+36 \mathrm{yrs}$ \\
\hline \hline
\end{tabular}




\section{An Oort cloud Fly-Through Mission}

Beyond the Heliopause the next scientifically notable destination is the solar gravitational lens at $550 \mathrm{AU}$. However, due to the need to maintain spacecraft position within $100 \mathrm{~m}$ of the gravitational lens in-order to exploit it and the inability of a solar sail propulsion system to slowdown a spacecraft within the outer solar system this scientifically notable destination is not considered suitable for outer solar system exploration using solar radiation pressure as the sole means of propulsion. The next scientifically notable destination is the Oort cloud (alternatively the Öpik-Oort Cloud), although it is noted that no direct observations have been made to validate its existence. The Oort cloud is thought to extend from approximately $50-200,000$ astronomical units, or $0.79-3.16$ light years, although an inner Oort cloud is also thought to possibly exist from two to twenty thousand astronomical units. The spherical shell of the Oort cloud is the ultimate boundary of the solar system, the edge of the sun's gravitational influence. Hence, the outer regions of the Oort cloud are only loosely bound to the solar system and are thus easily affected by the gravitational pull of passing stars and their own Oort cloud, occasionally dislodging comets from their orbits within the cloud and sending them towards the inner solar system as long-period comets. Assuming a isotropic and homogeneous cloud with a mean number density of between 1 and $10 \mathrm{AU}^{-3}$, the number of Oort cloud objects encountered was considered for a 30 year cruise (considered the upper reasonable limit) or 100-year cruise (considered the absolute upper limit) to $50000 \mathrm{AU}$. As the spacecraft travels through the Oort cloud $\mathrm{N}$ objects would be detected within a radius $\mathrm{R}$ of the spacecraft, in time $\mathrm{t}$. The volume of this cylinder through the Oort cloud is $\left[\pi \mathrm{R}^{2} v_{\propto} \mathrm{t}\right]$ so the detection rate $\mathrm{N} / \mathrm{t}$ can be obtained from,

$$
\mathrm{N}=\pi \mathrm{R}^{2} v_{\infty} \mathrm{t} \rho
$$

Conversely, the search distance from the spacecraft to enable a desired detection rate is given by,

$$
\mathrm{R}=\sqrt{\left(\frac{\mathrm{N}}{\mathrm{t}}\right) \frac{1}{\pi v_{\infty} \rho}}
$$

The results of this analysis for a 100-year cruise to the Oort Cloud show that 1 object per year may be detected within 200-600 Earth radii of the spacecraft, while for a faster 30 year cruise, 1 object per year may be detected within a closer range of 100-300 Earth Radii of the spacecraft. As such an Oort cloud mission can be expected to encounter a sufficient number of objects to be scientifically interesting. 
Oort cloud exploration is clearly an extremely challenging goal, especially when using solar radiation pressure alone to propel the spacecraft. Transit to such a large heliocentric distance necessitates either a long duration mission of order 100 years or more, or extremely high cruise speeds to provide reasonable mission durations. Considering a 100-year cruise to the Oort cloud requires the spacecraft to maintain a velocity of $500 \mathrm{AU} / \mathrm{yr}$, while a 30 year cruise, requires the cruise speed to be increased to approximately $1666 \mathrm{AU} / \mathrm{yr}$ ( $2.5 \%$ of light speed). These are clearly immense requirements to be imposed on the propulsion system.

Returning to the concept of the close solar photonic assist, the closer the perihelion of the trajectory, the greater the photon flux and so thrust produced by the solar sail. However, the minimum distance from the sun, which from the IHP study was seen to be critical, is dependent on the thermal properties of the sail. The use of aluminum as a reflective layer limits the close-approach radius. Hence, alternative approaches are sought such as the use of Beryllium or an all-carbon mesh sail. The use of carbon reduces the sail mass, due to the low density of carbon, while extending the temperature envelope of the sail. It should be further noted that a carbon sail would be immune to ultra-violet degradation and does not cling or wrinkle, allowing simple elastic deployment from a rolled stowage configuration. Compared to polymer substrate sails, or an all-metal sail, carbon mesh sails would be thick and highly porous. All carbon sails could be manufactured from fibers or tubes, id est, carbon nanotubes. Carbon nanotubes can be generated using chemical vapor deposition (CVD) in which the decomposing of an organic gas over a substrate covered with metal catalyst particles produces nanotubes. The CVD technique allows for the production of nanotubes on a much larger scale and is potentially a key to their use for solar sail applications. Furthermore, the nanotube configuration could potentially be finely tuned it would allow a carbon nanotube sail to be optimized for specific wavelengths corresponding to peaks in the solar spectrum. Thermally, carbon mesh sails could survive temperatures up to $\sim 3000 \mathrm{~K}$ (the melting point of carbon is $3800 \mathrm{~K}$ ), before out-gassing becomes excessive and sail degradation becomes an issue. Note an element of out-gassing could be utilized to provide a propulsive push at perihelion although this would degrade sail performance for later multifunctional duties, such as a parabolic antenna. If all-carbon sails were utilized then high-performance carbon sails could approach as close as four solar radii to the sun's surface.

Bulk carbon has a reflectivity of 0.27 at a wavelength of $1000 \mathrm{~nm},{ }^{*}$ which is beyond the visible wavelength range. Noting that as wavelength is reduced the reflectivity typically reduces, for example bulk molybdenum has

\footnotetext{
${ }^{*}$ Taken from www.webelements.com
} 
reflectivity 0.5737 at wavelength $1000 \mathrm{~nm}$ and reflectivity 0.5667 at $546 \mathrm{~nm}$ (visible light) 60, an all carbon solar sail is estimated to have a reflectivity of order 0.25 ; significantly lower than an aluminum sail film. However, the increase in melting point allows a much closer solar approach radius and hence a much increased solar photonic assist. Use of doped carbon nanotubes, or a reflective coating can enhance reflectivity. However, carbon has the highest melting point of all the elements and hence any added reflective coating would reduce the sail closeapproach radius and would hence require a complex trade be performed. For example, tungsten has the second highest melting point of all elements, with the highest strength (of metals) at temperatures over $1900 \mathrm{~K}$ and has a reflectivity of 0.46 . Hence, a tungsten coating could notably increase sail reflectivity, however tungsten has a density of $19250 \mathrm{~kg} / \mathrm{m}^{3}$, and even a very thin tungsten layer would significantly increase sail mass offsetting any benefits of increased reflectivity. Furthermore, tungsten does not sublime.

The simplest way of reaching four solar radii is a direct launch to a JGA which can be used to target a low perihelion. However, the required launch $\mathrm{C}_{3}$ for a direct to Jupiter trajectory is of order $100 \mathrm{~km}^{2} / \mathrm{s}^{2}$, this could possibly be reduced to as low as $8 \mathrm{~km}^{2} / \mathrm{s}^{2}$ by using multiple inner planet gravity assists at the cost of extended mission duration. Assuming a direct to JGA launch the minimum solar approach is achieved in 3.7 years. The allcarbon sail will be stowed until the minimum solar approach to minimize sail performance degradation.

An analysis of the cruise speed attained as a function of the solar sail lightness number was performed. A lightness number of 1 corresponds to a characteristic acceleration of $5.93 \mathrm{~mm} / \mathrm{s}^{2}$. If a lightness number of 1 is used and the sail is pitched face on to the sun, then solar gravity is effectively "switched off" and the sail follows a rectilinear path, with the perihelion velocity conserved. For the Jupiter-solar perihelion transfer, an ellipse with an aphelion at 5.2 AU (Jupiter) and a perihelion at $0.0186 \mathrm{AU}$ (4 solar radii) was considered. For a lightness number of 1, the rectilinear post-perihelion trajectory will be at $90^{\circ}$ to the incoming ellipse. The perihelion velocity can be determined as 65.04 AU/year, with the spacecraft reaching the $200 \mathrm{AU} 3.08$ years post-perihelion, $1000 \mathrm{AU}$ in 15.4 years and the Oort cloud in 768 years. This mission duration is clearly unacceptable and so the characteristic acceleration needs to be increased to far in excess of $5.93 \mathrm{~mm} / \mathrm{s}^{2}$. For lightness numbers greater than 1 , the trajectory is an inverted hyperbola (net force of the sail is outwards). Considering the conservation of specific energy, from perihelion to the cruise speed, $\mathrm{v}_{\infty}$, the cruise speed can be obtained by as, 


$$
\mathrm{v}_{\infty}=2 \pi \sqrt{\frac{2 \mathrm{r}_{\mathrm{a}}}{\mathrm{r}_{\mathrm{p}}^{2}+\mathrm{r}_{\mathrm{p}} \mathrm{r}_{\mathrm{a}}}-\frac{2(1-\beta)}{\mathrm{r}_{\mathrm{p}}}} \text { AU/year }
$$

From (5) it is determined that a 100-year cruise to the Oort cloud is enabled with a characteristic acceleration of 343 $\mathrm{mm} / \mathrm{s}^{2}$, while a faster 30 year cruise would be possible using a characteristic acceleration of $3874 \mathrm{~mm} / \mathrm{s}^{2}$.

Selecting the "fast" mission as baseline and with the required sail performance defined the sail loading (total spacecraft mass per unit area) required for a sail characteristic acceleration of $3874 \mathrm{~mm} / \mathrm{s}^{2}$ is $0.59 \times 10^{-3} \mathrm{~g} / \mathrm{m}^{2}$. Designing for a launch $\mathrm{C}_{3}$ of $100 \mathrm{~km}^{2} / \mathrm{s}^{2}$, the Ariane $5 \mathrm{ECB}$ with multiple ignition (Ariane V Versatile) can deliver approximately $750 \mathrm{~kg}$ to this launch energy, the total mission launch mass is thus defined as $675 \mathrm{~kg}$, allowing a $10 \%$ launch mass margin. The launch mass will consist of flight and non-flight mass (deployer mass), therefore assuming the non-flight mass is approximately $200 \%$ of the flight mass then the flight mass is $250 \mathrm{~kg}$, which requires a vast disc sail of radius $11629 \mathrm{~m}$.

The available non-sail mass fraction was assumed as $10 \%$ of the flight mass. Therefore, the non-sail mass will have an allocation of order $25 \mathrm{~kg}$, which will be evenly distributed over the entire sail surface and not as a centrally located bus, necessitating a bus areal density of less than $59 \mu \mathrm{g} / \mathrm{m}^{2}$. From the allocated $25 \mathrm{~kg}$ all the normal subsystems such as telecommunications, command and data handling and power must be provided. Additionally, a remote sensing capability, such as a Radar (RAdio Detection And Ranging) or LIDAR (Light Detection And Ranging) system is required, with molecular electronics and micro-machining providing early indications of how such systems could be developed. The bus systems should be incorporated into the sail film, with small micro-nodes placed at the intersections of the sail film grid network. The nodes will communicate with each other using the quantum conductance of the carbon nanotube sail, which will also generate the on-board power by utilizing the inductive effect of the motion of the conducting sail through the interstellar magnetic field with power storage achieved by tuning some of the carbon nanotubes to act as semi-conductor storage devices. The sail will be able to generate almost $10^{-7} \mathrm{~W} / \mathrm{m}^{2}$ by such means, assuming an interstellar magnetic field strength of order $10^{-10} \mathrm{~T}$.

Communications at path lengths beyond $1000 \mathrm{AU}$ necessitate the use of non-RF systems due to the significant space losses incurred; as such an optical system is required. Noting that the divergence of a Gaussian Beam (laser) is larger for longer wavelengths a laser wavelength of $890 \mathrm{~nm}$ (near infra-red) was assumed. Considering a distributed antenna diameter matching that of the sail, a phased array laser transmitter working as a coherent light source was assumed. The optical system will communicate with a near-Earth relay spacecraft, with terminal antenna of $6.5 \mathrm{~m}$, 
which is the preferred option as it eliminates atmospheric losses in the signal. Assuming that approximately $10 \%$ of the available power is used for telecommunications purposes, an upper limit of $10^{-8} \mathrm{~W} / \mathrm{m}^{2}$ of power is available. It was thus found that the vast aperture provides a data rate of order $4 \mathrm{M} \mathrm{bps}$ at $200000 \mathrm{AU}$.

The Oort cloud mission timeline for a 30 year ("fast") and 100 year ("slow") cruise are detailed in Table 7 . Table 7 Oort Cloud mission timeline from sail deployment

\begin{tabular}{llll}
\hline \hline Solar Distance (AU) & Milestone & "Slow" Mission & "Fast" Mission \\
\hline 30 & Kuiper Belt & 3.2 weeks & 6.6 days \\
50 & & 1.2 months & 11 days \\
100 & & 2.4 months & 3.1 weeks \\
200 & Heliopause & 4.8 months & 1.4 month \\
1000 & & 2.0 years & 7.2 months \\
50,000 & Inner edge of Oort cloud & 100 years & 30 years \\
200,000 & Outer edge of Oort Cloud & 400 years & 120 years \\
\hline \hline
\end{tabular}

\section{Discussion of Solar Sail Technology Development}

Technology can be developed following two primary models either technology push or applications pull. For space systems engineering, a technology is typically developed and adopted once a clear enabling capability has been identified to justify the development expenditure. The end-point of solar sailing applications pull was investigated through consideration of exploration beyond the Kuiper-Belt. To realize the end-point of solar sailing applications pull the identified technology requirements of that end-point application must be enabled through prior applications, with each prior mission acting as a stepping stone to the next. It is of note that the first solar sail mission application will likely be required to overreach the required technology for that application in isolation; however, the mission would require to be considered with a larger roadmap context 31,34 .

Using solar radiation pressure alone to propel the spacecraft, no impossibility was identified when considering an Oort cloud mission. However, the practical difficulties of the mission, such as control of a near-twelve kilometer structure, means that alternative approaches are likely to be sought. An Oort cloud mission is thus classed as beyond far-term for solar sail propulsion and hence unsuitable as a solar sailing applications pull. With this, it is considered that the IHP mission is the end-point of solar sailing applications pull. Thus, the enabling solar sail technology requirements of the IHP mission application must be devolved to preceding missions such that the IHP mission is 
enabled through a coherent development roadmap. Future technology development of solar sailing is required to enable these technology requirements if a solar sail IHP mission application is to be enabled. As such, the current solar sail technology development focus on 3-axis stabilized square solar sails is not fully compatible with any future solar sail IHP mission application; however, a Kuiper Belt mission does appear feasible. The development of solar sailing technology thus appears to be at an important juncture, where either current technology activities are revisited to enable an application such as the IHP mission, perhaps through a hybrid sail architecture, say, a spinning square sail. Or, it is accepted that solar sailing will likely never be used for such a mission. However, accepting the latter critically limits the number of applications pulling solar sail technology development and hence critically questions the sustainability of future solar sail technology development within the current 3-axis stabilized square sail architecture.

\section{Conclusions}

The use of solar sail propulsion has been considered for exploration of the Kuiper Belt and beyond. A detailed analysis of a 25-year Interstellar Heliopause Probe mission was conducted using a method of integrated statistical regression analysis to identify critical, enabling technology requirements. It was found that the 25-year mission duration necessitated a solar sail characteristic acceleration of $1.5 \mathrm{~mm} / \mathrm{s}^{2}$. A very large3-axis stabilized square solar sail, or a spinning disc sail with assembly loading of $1.8 \mathrm{~g} / \mathrm{m}^{2}$, which is within the range of prior spinning sail design concepts, is thus required. However, significant sail technology development in areas such as sail film areal density reduction is required. Furthermore, non-solar sail critical spacecraft systems were identified in-order to minimize the mission Advancement Degree of Difficulty by reducing the technology requirements on the solar sail. It was found that a Radioisotope Thermoelectric Generators power density of approximately $12 \mathrm{~W} / \mathrm{kg}$ was required to enable the mission. However, it was also found that a power density above this is not required as it provides only modest benefits for the sail sizing. Similarly, an advanced lightweight composite High-Gain Antenna and a high-efficiency Ka-band travelling-wave tube amplifier were found to be critical enabling technologies. Furthermore, as the sail structure is likely to be jettisoned at $5 \mathrm{AU}$ for science reasons, a reliable concept for sail separation is needed, as is a similar concept to separate the sail from its deployment module. Finally, autonomous operation beyond Jupiter and during the science phase is an important requirement to reduce ground segment costs for such a long duration mission. 
Following the Interstellar Heliopause Probe mission a similar Kuiper Belt mission study was briefly considered which would act as a pre-curser. It was found that the Kuiper Belt mission could be conducted with a sail assembly loading of $3.3 \mathrm{~g} / \mathrm{m}^{2}$ and a sail of radius $63 \mathrm{~m}$. A low-cost Rockot launch vehicle was found to be suitable for this mission. It was also noted that the Kuiper Belt mission could be performed by a 3-axis stabilized square sail of side length $130 \mathrm{~m}$ and assembly loading $6.5 \mathrm{~g} / \mathrm{m}^{2}$, however such a mission could not easily act as a technology pathfinder towards a Heliopause mission due to the required sail performance of the later mission.

Finally, an Oort cloud mission study was presented where it was noted that beyond the Heliopause the Oort cloud is the next scientifically interesting destination available by solar sail propulsion. The Oort cloud mission was found to require a significant increment in technology capabilities beyond the Interstellar Heliopause Probe mission, with a very close solar pass, an all-carbon mesh sail and a distributed spacecraft system across the sail surface generating power from the interstellar magnetic field. In conclusion, a solar sail Oort cloud mission is considered beyond far-term technology. As such, the Interstellar Heliopause Probe mission is considered the outer most mission scenario from our solar system which will likely use solar sail technology and the likely goal point of any solar sail technology development program. Therefore, the conclusion that a spinning sail is required, contrary to previous studies, for any reasonable Interstellar Heliopause Probe mission is critical as this technology requirement must be enabled through prior missions, with each mission acting as an enabling facilitator towards the next mission. Note that it is highly unlikely that, say, a 3-axis stabilized square sail mission to the Kuiper Belt will provide much, if any, confidence to then progress to a spin-stabilized disc solar sail architecture for an Interstellar Heliopause Probe mission. As such, for a far-term solar sail mission such as an Interstellar Heliopause Probe to be enabled all preceding missions must act as enabling facilitators to the next mission and must therefore develop the sail architecture required for such far-term missions. With this conclusion, the on-going and future technology development of square solar sails should be carefully considered.

\section{References}

1. McInnes, C.R., "Solar Sailing: Technology, Dynamics and Mission Applications", Springer-Praxis, Chichester, ISBN 1-85233-102-X, 1999.

2. West, J.L., "Design Issues for a Mission to Exploit the Gravitational Lensing Effect at 500 AU", $2^{\text {nd }} I A A$ Symposium on Realistic Near-term Advanced Scientific Space Missions, Aosta, Italy, June 1998. 
3. Leipold, M., Wagner, O., "Solar Photonic Assist Trajectory Design for Solar Sail Missions to the Outer Solar System and Beyond", Advances in the Astronautical Sciences, Vol. 100, No. 2, 1998, pp. 1035-1045.

4. Vulpetti, G., "Sailcraft at High Speed by Orbital Angular Momentum Reversal”, Acta Astronautica, Vol. 40, No. 10, 1997, pp. 733-758.

5. Sauer, C., "Solar Sail Trajectories for Solar Polar and Interstellar Probe Missions," Astrodynamics 1999, edited by K. Howell, F. Hoots, and B. Kaufman, Vol. 103 of Advances in the Astronautical Sciences, Univelt, Inc., 2000, pp. 547-562.

6. Yen, C. L., "Comparing Solar Sail and Solar Electric Propulsions for Propulsive Effectiveness in Deep Space Missions," American Astronautical Society, AAS Paper 01-214, Feb. 2001.

7. Sweetser, T. H., Sauer, C. G., “Advanced Propulsion Options for Missions to the Kuiper Belt,” AAS/AIAA Astrodynamics Specialists Conference, Quebec City, Canada, August, 2001.

8. Colasurdo, G., Casalino, L., “Optimal Control Law for interplanetary Trajectories with Solar Sail”, Advances in the Astronautical Sciences, Vol. 109, Pt. 3, 2001, pp. 2357-2368.

9. Vulpetti, G., "Sailcraft Trajectory Options for the Interstellar Probe: Mathematical Theory and Numerical Results”, Chapter IV of NASA/CR-2002-211730, “The Interstellar Probe (ISP): Pre-Perihelion Trajectories and Application of Holography”, June 2002.

10. Dachwald, B., "Interplanetary Mission Analysis for Non-Perfectly Reflecting Solar Sailcraft Using Evolutionary Neurocontrol", Advances in the Astronautical Sciences, Vol. 116, Suppl. 2004, pp. 1-18.

11. Sharma, D.N., Scheeres, D.J., "Solar System Escape Trajectories Using Solar Sails “, Journal of Spacecraft and Rockets, Vol. 41. No. 4, 2004, pp. $684-687$.

12. Dachwald, B., "Solar Sail Performance Requirements for Missions to the Outer Solar System and Beyond", IAC-04-S.P.11, Proceedings of the $55^{\text {th }}$ International Astronautical Congress of the International Astronautical Federation, the International Academy of Astronautics, and the International Institute of Space Law, Vancouver, Canada, October 2004.

13. Dachwald, B., "Optimal Solar-Sail Trajectories for Missions to the outer Solar System”, Journal of Guidance, Control and Dynamics, Vol. 28, No. 6, 2005, pp. $1187-1193$.

14. Macdonald, M., M드ees, C.R., Dachwald, B., "Heliocentric Solar Sail Orbit Transfers with Locally Optimal Control Laws”, Journal of Spacecraft and Rockets, Vol. 44. No. 1, 2007, pp. 273 - 276. 
15. Leipold, M., Fichtner, H., Heber, B., Groepper, P., Lascar, S., Burger, F., Eiden, M., Niederstadt, T., Sickinger, C., Herbeck, L., Dachwald, B., Seboldt, W., "Heliopause Explorer - A Sailcraft Mission to the Outer Boundaries of the Solar System”, Acta Astronautica, Vol. 59, 2006, pp. 785 - 796.

16. Mc Innes, C.R., "Delivering Fast and Capable Missions To The Outer Solar System", Advances in Space Research, Vol. 34, Iss. 1, 2004, pp. $184-191$.

17. Matloff, G.L., "Interstellar Solar Sailing: Comparison of Five Candidate Sail Materials”, IAA-99-IAA.4.1.09, The $50^{\text {th }}$ International Astronautical Congress, Amsterdam, The Netherlands, October 1999.

18. Kluever, C.A., "Heliospheric Boundary Exploration Using Ion Propulsion Spacecraft”, Journal of Spacecraft and Rockets, Vol. 34. No. 3, 1997, pp. $365-371$.

19. Bramanti, C., Izzo, D., Samaraee, T., Walker, R., Fearn, D., "Very high delta-V missions to the edge of the solar system and beyond enabled by the dual-stage 4-grid ion thruster concept", Acta Astronautica, Vol. 64, 2009 , pp. $735-744$.

20. McNutt Jr., R.L., Andres, G.B., McAdams, J., Gold, R.E., Santo, A., Oursler, D., Heeres, K., Fraeman, M., Williams, B., “Low-cost interstellar probe”, Acta Astronautica, Vol. 52, 2003, pp. 267 - 279.

21. McNutt Jr., R.L., Andrews. G.B., Gold, R.E., Bokulic, R.S., Boone, B.G., Haley, D.R., McAdams, J.V., Williams, B.D., Boyle, M.P., Starstrom, G., Riggin, J., Lester, D., Lyman, R., Ewing, M., Krishnan, R., Read, D., Naes, L., McPherson, M., Deters, R., “A realistic interstellar explorer”, Advances in Space Research, Vol. 34, 2004, pp. $192-197$.

22. Zurbuchen, T.H., Prashant, P., Gallimore, A., Scheeres, D., Murphy, N., Zank, G., Malhotra, R., Funsten, H., “Interstellar Probe: Breakthrough Science Enabled by Nuclear Propulsion”, IAC-04-IAA.3.6.4.08, Electronic Proceedings of the $55^{\text {th }}$ International Astronautical Congress of the International Astronautical Federation, the International Academy of Astronautics, and the International Institute of Space Law, Vancouver, Canada, October 2004.

23. Fiehler, D.I., McNutt Jr., R.L., "Mission Design for the Innovative Interstellar Explorer Vision Mission", NASA Report NASA/CR-2005-214017, November 2007.

24. Fiehler, D.I., $\mathbf{M}^{\mathrm{c}}$ Nutt Jr., R.L., "Mission Design for the Innovative Interstellar Explorer Vision Mission”, Journal of Spacecraft and Rockets, Vol. 43. No. 6, 2006, pp. 1239 - 1247. 
25. Wallace, R.A., "Precursor Missions to Interstellar Exploration", Proceedings of Aerospace Conference 1999, Vol. 1, 1999, pp. 413 420, ISBN: 0-7803-5425-7.

26. Wallace, R.A., Ayon, J.A., Sprague, G.A., "Interstellar Probe Mission/System Concept", Proceedings of Aerospace Conference 2000, Vol. 7, 2000, pp. 385 - 396, ISBN: 0-7803-5846-5.

27. Garner, C.E., Layman, W., Gavit, S.A., Knowles, T., “A Solar Sail design For A Mission To The Interstellar Medium", Proceedings of "Space Technology and Applications International Forum”, Edited by M. El-Genk, AIP Conference Proceedings 504, NY, 2000, pp. 947-961.

28. Lyngvi, A., Falkner, P., Peacock, A., "The Interstellar Heliopause Probe”, Tools and Technologies for Future Planetary Exploration, 37 $7^{\text {th }}$ ESLAB Symposium, ESTEC, 2003.

29. Lyngvi, A., Falkner, P., Kemble, S., Leipold, M., Peacock, A., "The Interstellar Heliopause Probe", Acta Astronautica, Vol. 57, 2005, pp. $104-111$.

30. Lyngvi, A., Falkner, P., Peacock, A., "The Interstellar Heliopause Probe Technology Reference Study", Advances in Space Research, Vol. 35, 2005, pp. 2073 - 2077.

31. Macdonald M., M'Innes C. R., “A Near-Term Roadmap for Solar Sailing”, IAC-04-U.1.09, Electronic Proceedings of the $55^{\text {th }}$ International Astronautical Congress of the International Astronautical Federation, the International Academy of Astronautics, and the International Institute of Space Law, Vancouver, Canada, October 2004.

32. Macdonald, M., Hughes, G., McInnes, C. R., Lyngvi, A., Falkner, P., Atzei, A., "Solar Polar Orbiter: A Solar Sail Technology Reference Study”, Journal Spacecraft \& Rockets, Vol. 43, No. 5, 2006, pp. 960-972.

33. Bilbro, J.W., "Systematic Assessment of the Program/Project Impacts of Technological Advancement and Insertion”, George C. Marshall Space Flight Center, Dec. 2006. Available from, http://lincoln.gsfc.nasa.gov/trl/Bilbro2006.pdf cited 18 February 2010.

34. Macdonald, M., Hughes, G.W., M'Innes, C. R., Lyngvi, A., Falkner, P., Atzei, A., "GeoSail: An Elegant Solar Sail Demonstration Mission”, Journal Spacecraft and Rockets, Vol. 44, No 4, 2007, pp 784 - 796.

35. Gurnett, D. A., Kurth, W. S., Allendorf, S. C., and Poynter, R. L., "Radio Emission from the Heliopause Triggered by an Interplanetary Shock,” Science, Vol. 262 (5231), October 1993, pp. 199-203. 
36. Axford, W. I., "The Interaction of the Solar Wind with the Interstellar Medium" from Chapter 9 of Solar Wind, NASA SP-308, Edited by Sonett, C.P., Coleman, Jr., P. J., and Wilcox, J. M., NASA, Washington D.C., 1972, pp 609-657.

37. Linsky, J. L., Wood, B. E., “The Alpha Centauri Line of Sight: D/H Ratio, Physical Properties of Local Interstellar Gas, and Measurement of Heated Hydrogen (The 'Hydrogen Wall') Near the Heliopause", Astrophysical Journal, Vol. 463, 1996, pp. 254-270.

38. Herbeck, L., Leipold, M., Sickinger, C., Eiden, M., Unckenbold, W., "Development and Test of Deployable Ultra-Lightweight CFRP-Booms for a Solar Sail”, Proceedings of the European Conference on Spacecraft Structures, Materials and Mechanical Testing, Edited by C. Stavrinidis, A. Rolfo, and E. Breitbach. European Space Agency, ESA SP-468, 2001., pp.107 - 112.

39. Herbeck, L., Sickinger, C., Eiden, M., Leipold, M., "Solar Sail Hardware Developments", European Conference on Spacecraft Structures, Materials and Mechanical Testing, Toulouse 2002.

40. Leipold, M., Eiden, M., Garner, C. E., Herbeck, L., Kassing, D., Niederstadt, T., Krüger, T., Pagel, G., Rezazad, M., Rozemeijer, H., Seboldt, W., Schöppinger, C., Sickinger, C., Unckenbold, W., "Solar Sail Technology Development and Demonstration", Acta Astronautica, Vol. 52, Iss. 2-6, 2003, pp. 317 - 326.

41. McEachen, M.E., Trautt, T.A., Murphy, D.M., “The ST8 SAILMAST Validation Experiment”, AIAA 20051884, 46th AIAA/ASME/ASCE/AHS/ASC Structures, Structural Dynamics \& Materials Conference, Austin, U.S.A. 2005.

42. Murphy, D.M., McEachen, M.E., Macy, B.D., "Demonstration of a 20-m Solar Sail System”, AIAA 20052126, 46th AIAA/ASME/ASCE/AHS/ASC Structures, Structural Dynamics \& Materials Conference, Austin, U.S.A. 2005.

43. Lichodziejewshi, D., Derbès, B., "Vacuum Deployment and Testing of a 4-Quadrant Scaleable Inflatable Rigidizable Solar Sail System", AIAA 2005-2122, 46th AIAA/ASME/ASCE/AHS/ASC Structures, Structural Dynamics \& Materials Conference, Austin, U.S.A. 2005.

44. Sleight D.W., Michii, Y., Lichodziejewshi, D., Derbès, B., Mann, T.O., "Structural Analysis of an Inflation Deployment Solar Sail with Experimental Validation”, AIAA 2005-3727, 41st AIAA/ASME/SAE/ASEE Joint Propulsion Conference \& Exhibit, Tucson, U.S.A. 2005. 
45. Mann, T., Behun, V., Lichodziejewshi, D., Derbès, B., Sleight, D., "Ground Testing a 20-meter Inflation Deployed Solar Sail”, AIAA 2006-1707, 47th AIAA/ASME/ASCE/AHS/ASC Structures, Structural Dynamics \& Materials Conference, Newport, U.S.A. 2006.

46. Young, R.M., Montgomery, E.E., Adams, C.L., "TRL Assessment of Solar Sail Technology Development Following the 20-meter System Ground Demonstrator Hardware Testing”, AIAA 2007-2248, 48th AIAA/ASME/ASCE/AHS/ASC Structures, Structural Dynamics \& Materials Conference, Honolulu, U.S.A. 2007.

47. Murphy, D.M., "Validation of a Scaleable Solar Sailcraft System", Journal of Spacecraft and Rockets, Vol. 44, No. 4, 2007, pp. $797-808$.

48. Kawaguchi, J., Mimasu, Y., Mori, O., Funase, R., Yamamoto, T., Tsuda, Y., "IKAROS - Ready for Lift-Off as the World's First Solar Sail Demonstration in Interplanetary Space", IAC-09-D1.1.3, Electronic Proceedings of the $60^{\text {th }}$ International Astronautical Congress of the International Astronautical Federation, the International Academy of Astronautics, and the International Institute of Space Law, Daejeon, South Korea, October 2009.

49. Dachwald, B., Macdonald, M., M'Innes, C. R., Mengali, G., Quarta, A.A., "Impact of Optical Degradation on Solar Sail Mission Performance", Journal Spacecraft and Rockets, Vol. 44, No 4, 2007, pp 740 - 749.

50. Gill, P. E., Murray, W., and Saunders, M. A., "SNOPT: An SQP Algorithm for Large-Scale Constrained Optimization,” SIAM Journal on Optimization, Vol. 12, No. 4, 2002, pp. 979-1006.

51. Macdonald M., M'Innes C. R., "Analytical Control Laws for Planet-Centred Solar Sailing”, Journal of Guidance, Control, and Dynamics, Vol. 28, No. 5, 2005, pp. 1038-1048.

52. Garrett, H.B., Jun, I., Shapiro, A.A., "Interstellar Space Missions: Ultra-Reliability Requirements and Engineering Issues", presented at the 45th AIAA Aerospace Sciences Meeting and Exhibit, Reno, U.S.A., January 2007.

53. Garrett, H.B., Shapiro, A.A., Yang, J.Y., "Interstellar Space Missions: Ultra-Reliability Requirements and Engineering Issues - Part II", presented at the 46th AIAA Aerospace Sciences Meeting and Exhibit, Reno, U.S.A., January 2008.

54. Wright, J. L., Space Sailing, Gordon \& Breach Science Publishers, Amsterdam, 1992, ISBN 2-88124-842-X. 
55. Rogan, J., Gloyer, P., Pedlikin, J., Veal, G., Derbes, B., "Encounter 2001: Sailing to The Stars”, SSC01-112, $15^{\text {th }}$ Annual/USU Conference on Small Satellites, 2001.

56. Salama, M., White, C., Leland, R., "Ground Demonstration of a Spinning Solar Sail Deployment Concept,” Journal of Spacecraft and Rockets, Vol. 40, No.1, 2003, pp. 9-14.

57. "Space Mission Analysis and Design (Space Technology Library)", Edited by Wertz, J.R. and Larson, W.J., Third Edition, Microcosm and Kluwer, New York, ISBN-13: 978-0792359012, 1999.

58. Williams, D.R., “Jupiter Fact Sheet”, NSSDC Planetary Fact Sheets, NASA Goddard, Available from URL: http://nssdc.gsfc.nasa.gov/planetary/factsheet/jupiterfact.html, 2007.

59. Hills, J.G., "Comet Showers and the Steady-State Infall of Comets from the Oort Cloud", The Astronomical Journal 86: 1730-1740. doi:10.1086/113058, 1981.

60. “American Institute of Physics (AIP) Handbook”, New York: McGraw-Hill, $3^{\text {rd }}$ ed., edited by Gray, Dwight E, 1972. 